\title{
Electrospun Fibers for Drug Delivery after Spinal Cord Injury and the Effects of Drug Incorporation on Fiber Properties
}

\author{
Christopher D.L. Johnson Anthony R. D'Amato Ryan J. Gilbert \\ Department of Biomedical Engineering, and Center for Biotechnology and Interdisciplinary Studies, \\ Rensselaer Polytechnic Institute, Troy, N.Y., USA
}

\section{Key Words}

Electrospun fibers · Drug incorporation · Riluzole .

Neurotrophin-3 · Spinal cord injury · Fiber characteristics .

Alignment · Diameter

\begin{abstract}
There is currently no cure for individuals with spinal cord injury $(\mathrm{SCl})$. While many promising approaches are being tested in clinical trials, the complexity of $\mathrm{SCl}$ limits several of these approaches from aiding complete functional recovery. Several different categories of biomaterials are investigated for their ability to guide axonal regeneration, to deliver proteins or small molecules locally, or to improve the viability of transplanted stem cells. The purpose of this study is to provide a brief overview of $\mathrm{SCl}$, present the different categories of biomaterial scaffolds that direct and guide axonal regeneration, and then focus specifically on electrospun fiber guidance scaffolds. Much like other polymer guidance approaches, electrospun fibers can retain and deliver therapeutic drugs. The experimental section presents new data showing the incorporation of two therapeutic drugs into electrospun poly-L-lactic acid fibers. Two different concentrations of either riluzole or neurotrophin-3 were loaded into the electrospun fibers to examine the effect of drug concen-
\end{abstract}

C.D.L.J. and A.R.D. contributed equally to this article. tration on the physical characteristics of the fibers (fiber alignment and fiber diameter). Overall, the drugs were successfully incorporated into the fibers and the release was related to the loading concentration. The fiber diameter decreased with the inclusion of the drug, and the decreased diameter was correlated with a decrease in fiber alignment. Subsequently, the study includes considerations for successful incorporation of a therapeutic drug without changing the physical properties of the fibers.

(c) 2016 S. Karger AG, Basel

\section{Abbreviations used in this paper}

\section{AN 6-aminonicotinamide}

BDNF brain-derived neurotrophic factor

BSA bovine serum albumin

ChABC chondroitinase ABC

CNS central nervous system

DMSO dimethyl sulfoxide

GDNF glial cell-derived neurotrophic factor

HAMC hyaluronan and methylcellulose

HFP 1,1,1,3,3,3-hexafluoro-2-propanol

NT-3 neurotrophin-3

PLGA poly-L-lactic-co-glycolic acid

PLLA poly-L-lactic acid

SCI spinal cord injury

SEM scanning electron microscopy

\section{KARGER}

(c) 2016 S. Karger AG, Basel

E-Mail karger@karger.com

www.karger.com/cto
Ryan J. Gilbert, PhD

Department of Biomedical Engineering

1108 th Street

Troy, NY 12180-3590 (USA)

E-Mail gilber2@ rpi.edu 


\section{Introduction: Concepts and Review of the Literature}

\section{Spinal Cord Injury}

\section{Demographics}

Spinal cord injury (SCI) is a devastating condition that leads to partial or total tetraplegia or paraplegia. The $\mathrm{Na}$ tional Spinal Cord Injury Statistical Center (NSCISC) at the University of Alabama at Birmingham reports that there are approximately 12,500 new SCI incidences every year in the USA. Improvements in medical care are increasing survival rates, which increases the financial burden on patients and their families. The financial burden is estimated to average between 1 and 5 million dollars in health care and treatment costs throughout the lifetime of the patient [NSCISC, 2014]. Thus, there is a need to develop new multifaceted approaches to achieve complete functional restoration.

\section{Pathophysiology}

Primary SCI occurs when a mechanical insult fractures the vertebral disc surrounding the soft tissue of the spinal cord. Bone fragments sever long axonal tracts at the site of injury and damage the neurons, oligodendrocytes, and astrocytes at the impact site. Additionally, mechanical insult at the injury site ruptures capillaries leading to an influx of blood circulating leukocytes. The release of several chemicals from the damaged cells and invading leukocytes create a chemical environment that is toxic to cells surviving the initial mechanical insult. This toxic environment leads to secondary SCI that causes the lesion to expand [Liu et al., 1997; Norenberg et al., 2004; Oyinbo, 2011]. Astrocytes surviving the initial injury become reactive from both the mechanical insult and chemokines released by the inflammatory cells. Reactive astrocytes change phenotypically by growing in size, proliferating at the lesion edge, and releasing axonal inhibiting chondroitin sulfate proteoglycans into the extracellular space [Sofroniew, 2009; Sofroniew and Vinters, 2010]. The reactive astrocytes, although helping to reform the blood-brain barrier and restrict leukocyte infiltration [Faulkner et al., 2004], form an environment inhospitable to the growth cones extending from regenerating axons into the lesion site [He et al., 2009; Gros et al., 2010; Fan et al., 2011]. Recent evidence suggests that it is not accurate to view reactive astrocytes and the glial scar as completely inhibitory to regenerating axons. Reactive astrocytes upregulate a number of genes associated with promoting neurite outgrowth (NG2, neuroglycan C, syndecan 1-4, FGF-2, glypican 1 and 5, and matrillin

Electrospun Fibers for Drug Delivery after SCI
2 ) and present laminin to guide regenerating axons [Anderson et al., 2016].

The axotomy of neurons within the white matter tracts severs an axon into a proximal segment and a distal segment. The distal axon segment is no longer connected to the soma of the neuron and breaks down in a process known as Wallerian degeneration [Bresnahan, 1978; Beattie et al., 2000; Norenberg et al., 2004]. The resulting debris from these axonal segments is cleared by macrophages. Oligodendrocytes surrounding distal segments die through apoptosis during Wallerian degeneration [Gros et al., 2010; Meinel et al., 2012]. Thus, the approach used to facilitate functional recovery should target specific cells at the appropriate time point following injury. In general, acute injury occurs in the first seconds to minutes after injury, secondary injury spans from minutes to weeks after injury, and chronic injury ranges from months to years after injury [Norenberg et al., 2004; Oyinbo, 2011]. If applied acutely, the approach would rescue neurons and glial cells from cell death, restrict the infiltration of inflammatory leukocytes, and suppress glial scar formation while providing guidance cues for regenerating axons. If applied chronically, the approach should counteract astrogliosis, initiate the regeneration of axons into and through the lesion site, and promote remyelination of these axons by remyelinating oligodendrocytes. Thus, the approach to treating either acute or chronic SCI will be multifaceted and involve many different strategies that work together.

While a successful treatment of SCI will involve many strategies working collectively, one promising area is developing biomaterials for SCI application. This article presents a brief description of the different types of biomaterial constructs for SCI. The sections presented subsequently are not meant to be comprehensive reviews but a general overview. For comprehensive reviews focusing on specific categories of biomaterials and providing a more thorough analysis of different biomaterial strategies under development for repair of the injured spinal cord, refer to Straley et al. [2010], Perale et al. [2011], Macaya and Spector [2012], Pakulska et al. [2012], Haggerty and Oudega [2013], Krishna et al. [2013], Schaub et al. [2015a] and Tsintou et al. [2015]. The following sections present different biomaterial approaches either as nondirectional (unable to direct the extension of regenerating axons) or directional, and the results of such approaches when employed within an animal model of SCI. Subsequent sections present electrospun fibers as a viable approach to support directed axonal regeneration, followed by a general discussion of the changes in the physical properties of the fibers when they are combined with a therapeutic drug. 
Fig. 1. Implantation of an HAMC hydrogel loaded with NT-3-releasing PLGA nanoparticles promoted axonal growth into the spinal compression injury lesion site in adult rats. a The compression lesion site was significantly smaller after implantation of NT-3-releasing HAMC hydrogel due to increased axonal regeneration. b Enlarged view of the boxed area in a which shows NF200-positive axons growing into the lesion site. c Compression lesion sites in animals that received NT-3free HAMC hydrogel did not show an increase in axonal extension compared to the SCI control group (d). This figure was reproduced from Elliott Donaghue et al. [2015] with permission from the Royal Society of Chemistry. Scale bars $=500 \mu \mathrm{m}$.
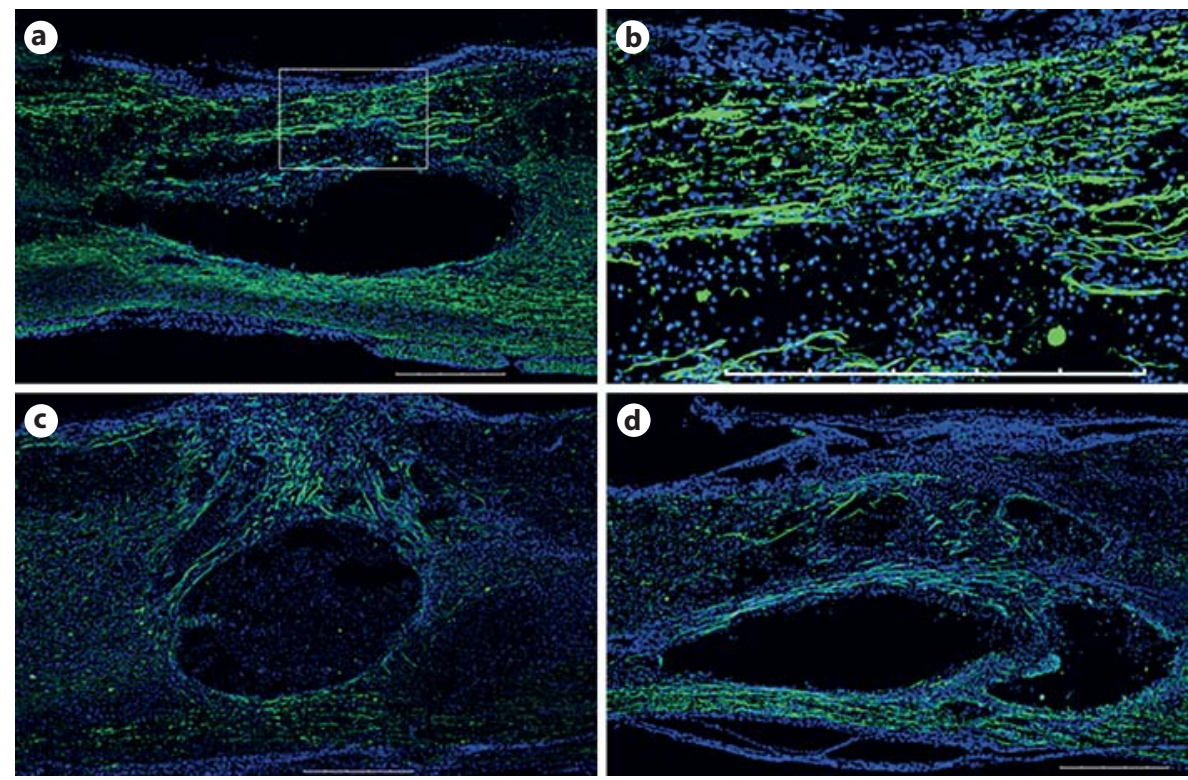

\section{Biomaterial Approaches Studied within Experimental Models of SCI}

The current biomaterial approaches developed to treat SCI can be divided into two categories: (1) approaches not meant to support directed axonal regeneration (known as nondirectional), and (2) approaches able to direct axonal regeneration through the lesion site (known as directional).

\section{Nondirectional Biomaterial Approaches}

The category of nondirectional biomaterial scaffolds can be divided into either hydrogels or composite biomaterials that consist of nanoparticles embedded within hydrogels. Hydrogel or hydrogel composites are typically isotropic, which makes it easier to design injectable scaffolds that can be delivered using minimally invasive techniques. Another benefit of these scaffolds is the capability to fine-tune their mechanical characteristics to match that of the spinal cord. Hydrogels can be manipulated to achieve tunable drug release through modification of the pore size or inclusion of ligands that can electrostatically retain the drug for extended release. However, the limitation of hydrogel scaffolds is their inability to provide directional guidance to regenerating axons attempting to traverse the SCI lesion site. Thus, their present application is to limit secondary injury and improve neuronal survival following injury.
As hydrogel scaffolds, by themselves, provide no directional cues for infiltrating cells, they are commonly fabricated to include small molecule pharmaceuticals to mitigate the severity of SCI. A figure published by Elliott Donaghue et al. [2015] (reproduced here as fig. 1) demonstrates the efficacy of hydrogels in animal models of SCI. Their particular hydrogel, a composite fabricated from hyaluronan and methylcellulose (HAMC) loaded with neurotrophin-3 (NT-3)-releasing nanoparticles, was injected into an acute compressive SCI model in adult rats. NT-3 release (fig. 1a) significantly reduced the lesion size when compared to lesion sizes observed when injured animals received an injection of drug-free HAMC hydrogels, or SCI controls not receiving biomaterial treatment (fig. 1c, d). Instead of employing a polysaccharide-based hydrogel, Taylor et al. [2006] placed a fibrin hydrogel within a rat suction ablation SCI model and observed the ability of fibrin hydrogel (without a drug) to promote axonal regeneration compared to animal controls not receiving the biomaterial treatment. The researchers developed an affinity-based system to slow the release of NT-3 from a fibrin hydrogel, and this system further enhanced the number and extension of axons into the lesion site. These studies and others employing hydrogels within rodent models of SCI require drugs to improve different SCI parameters (lesion size, axonal sprouting, etc.) [Taylor et al., 2004]. Results from Cox et al. [2015] provide additional validation that drug inclusion within hydrogels is necessary for the hydrogel approach 
Fig. 2. Implantation of templated agarose scaffold promoted the infiltration of axons into the spinal cord aspiration lesion site. a Control scaffolds promoted highly linear axonal extension through the scaffold's longitudinal channels. b Incorporation of bone marrow stromal cells (MSCs) increased axonal penetration into scaffolds compared to MSC-free control scaffolds. c Incorporation of brain-derived neurotrophic factor-secreting MSCs greatly increased axon penetration into the agarose scaffold compared to MSC loaded scaffolds and empty scaffolds. Scale bars $=100 \mu \mathrm{m}$. This figure was reproduced from Stokols et al. [2006] with permission from Mary Ann Liebert Inc.
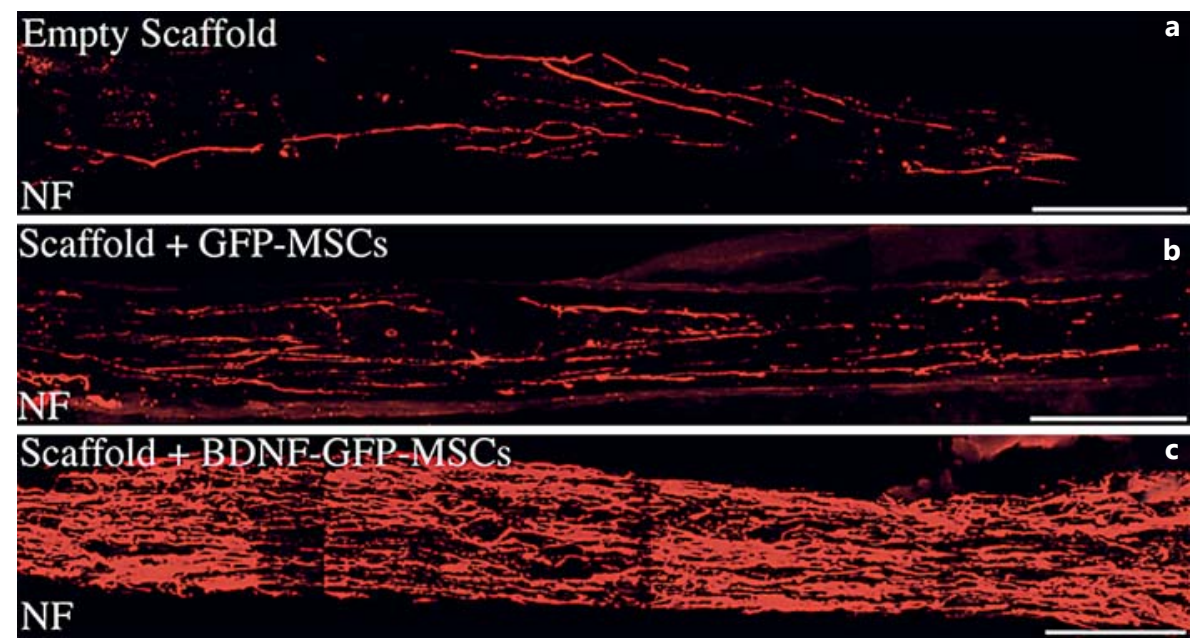

to robustly change the injury environment. Like the study presented by Elliott Donaghue et al. [2015], Cox et al. [2015] also used polymer spheres to extend the release of a drug from a polysaccharide hydrogel. Specifically, poly-L-lactic-co-glycolic acid (PLGA) nanoparticles containing 17- $\beta$ estradiol (an anti-inflammatory and neuroprotective steroidal hormone) were placed within an agarose hydrogel and the inflammatory response within a contusive rodent model was assessed. Control hydrogels containing drug-free nanoparticles upregulated multiple proinflammatory markers (IL-6, GRO-KC, MCP-1, and $S 100 \beta)$. However, animals that received hydrogels containing PLGA nanoparticles loaded with $17 \beta$-estradiol showed reduced expression of the abovementioned inflammatory markers. The hydrogel studies show that implanting hydrogels without therapy impedes (or only marginally improves) injury outcomes following SCI. However, secondary injury is generally reduced and/or axonal sprouting is improved. The secondary injury outcome depends on the type of drug incorporated into the hydrogel matrix.

\section{Directional Biomaterial Approaches}

With greater frequency, biomaterial scaffolds consisting of aligned pores or channels provide a substrate for directed axonal regeneration into and through the SCI environment. These scaffolds were created to recapitulate the organization of white matter tracts. One drawback is that it is difficult to design anisotropic materials that are injectable. Typically, materials with aligned topography are designed to be delivered as a conduit [He et al., 2009; Hurtado et al., 2011; Liu et al., 2012], which requires an invasive surgery that excises tissue in order to fit the regular geometry of the conduit. Early biomaterial attempts to provide directional cues for regenerating axons after SCI came in the form of polymer guidance conduits consisting of multiple aligned channels running longitudinally down the spinal cord. Several studies showed the ability of PLGA-aligned polymer scaffolds to increase axonal extension into the lesion site of a completely transected adult rat spinal cord [He et al., 2009; Fan et al., 2011]. Figure 2, which was originally published by Stokols et al. [2006], shows successful penetration and extension of axons into the channels within a templated agarose scaffold implanted into an aspiration model of SCI in adult rats. To further increase the rate and amount of axonal extension within these channels, the scaffolds were seeded with brain-derived neurotrophic factor (BDNF)producing cells prior to implantation. BDNF signaling promotes neuron survival [Acheson et al., 1995] and axon regeneration [Lindsay, 1988]. Inclusion of BDNF-releasing cells significantly increased axonal penetration and extension inside the scaffold.

It is a common approach to include neurotrophins in these polymer scaffolds. A collaborative effort between the Tuszynski and Sakamoto laboratories enhanced axonal infiltration into templated agarose conduits when incorporating two different neurotrophins (NT-3 and BDNF) [Stokols et al., 2006; Gros et al., 2010]. Other studies employing conduits with porous channels showed similar results. A study by Yao et al. [2013] implanted a porous collagen conduit with immobilized NT-3 genes which increased neuronal proliferation and extension in a completely transected rat spinal cord. Overall, these 


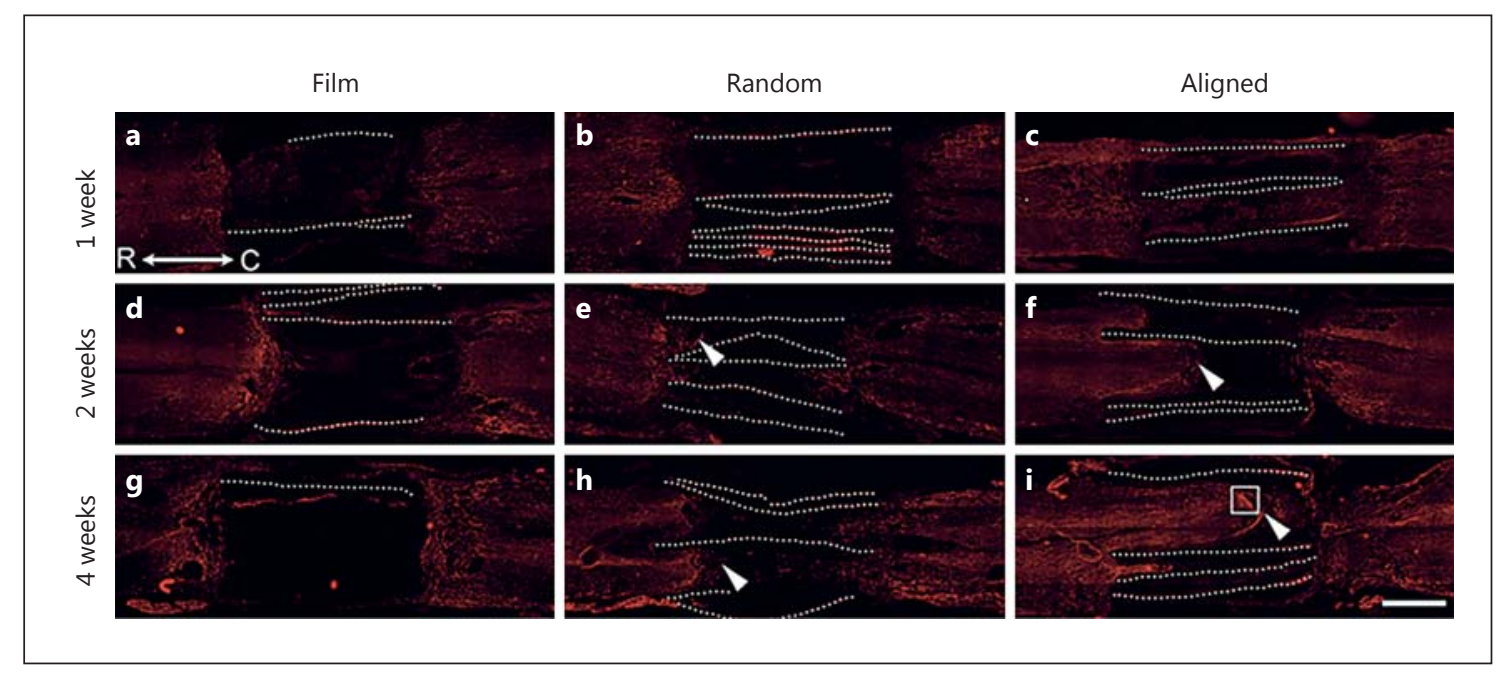

Fig. 3. Aligned electrospun PLLA fiber scaffolds implanted into a complete transection model of SCI in adult rats promote axonal regeneration across the lesion site. a, d, $\mathbf{g}$ PLLA films implanted into the SCI transection lesion did not allow for axonal extension into the lesion site over the course of 4 weeks. $\mathbf{b}, \mathbf{e}, \mathbf{h}$ Random fiber conduits promoted marginal axonal extension, but significantly less than the aligned fiber conduits in $\mathbf{c}, \mathbf{f}$, and i. Four weeks after the implantation of aligned fiber conduits, all animals exhibited axonal regeneration from the rostral spinal cord of at least $1.5 \mathrm{~mm}$. Scale bar $=1 \mathrm{~mm}$. This figure was reproduced from Hurtado et al. [2011] with permission from Elsevier. channels promote directed axonal extension in a limited manner into and through the lesion, but axonal regeneration was greatly enhanced when growth factors were included. Although these scaffolds provide important directional cues for axons after SCI, it is important to note that these biomaterials are only applied within transection or hemisection animal models of SCI due to their rigidity and larger size.

Similar to the polymer guidance conduits, fibrous constructs composed of electrospun fibers effectively enhance axonal regeneration into the lesion site. The physical characteristics of electrospun fibers can be tuned to create scaffolds with varying fiber alignment, chemistry, density, and/or diameter. In order to demonstrate the effects of alignment in vivo, a pioneering study performed by Hurtado et al. [2011] showed that a conduit of highly aligned electrospun poly-L-lactic acid (PLLA) fibers (of $1.5 \mu \mathrm{m}$ diameter) created a permissive environment for axonal extension and astrocytic migration into a complete transection rat model of SCI. The findings of this study are presented in figure 3 . Aligned electrospun PLLA fibers promoted significant axonal extension across a complete transection model of SCI in adult rats compared to axonal extension observed in polymer conduits containing randomly aligned fibers or polymer films (no fibers). Similarly, Liu et al. [2012] fabricated conduits composed of electrospun collagen fibers. These fibrous con- duits contained fibers with diameters of approximately $208 \mathrm{~nm}$ as opposed to the $1.5-\mu \mathrm{m}$ fibers seen in the previous study, and were implanted into a hemisection model of SCI in adult rats. These scaffolds promoted infiltration of regenerating axons and prevented the accumulation of astrocytes at the periphery of the injury. However, there seemed to be less infiltration of axons onto these smallerdiameter collagen scaffolds when compared to the largerdiameter PLLA scaffolds used by Hurtado et al. [2011].

Unlike the studies presented by Liu et al. [2012] and Hurtado et al. [2011], Zhu et al. [2010] immobilized the anti-inflammatory drug rolipram to the surface of PLLA/ PLGA electrospun fibers. Rolipram is a small-molecule drug that has shown a capability of reducing inflammation and promoting neurite outgrowth. After the implantation of these scaffolds in a rat hemisection model of SCI, robust regeneration was observed, and functional recovery after the implantation of drug-releasing scaffolds was significantly increased compared to the drug-free control fiber implantation. The authors also highlighted the ability of the drug-free fibers to decrease glial scar formation, and this reduction in glial scar was even more prevalent after the implantation of rolipram-containing fibers. In a study conducted by the Gelain laboratory, fibers (approximately $590 \mathrm{~nm}$ in diameter) delivered regenerative cytokines locally to a chronic postcontusive injured spinal cord. The treatment group showed significant regenera- 
tion of new central nervous system (CNS) tissue in the SCI lesion site, reduced inflammation, and reduced glial scarring compared to the control [Gelain et al., 2011]. In summary, conduits containing aligned fibers enabled robust axonal extension into the lesion site and, like channel-based strategies, benefit from the inclusion of a drug. To date only four studies have employed electrospun fibers within an animal model of SCI, and just two included a drug therapy. Thus, more study is necessary to understand the mechanisms by which aligned fibers promote regeneration. The next sections discuss the process of electrospinning and the challenges of drug inclusion within the fibers.

\section{Electrospun Fibers for Biological Applications}

In previous sections, aligned electrospun fibers promoted the regeneration of axons following acute SCI in experimental models [Hurtado et al., 2011]. Electrospun fiber scaffolds are studied more frequently in orthopedic applications, including tendon or ligament tissue engineering [Kumbar et al., 2008], and bone tissue engineering [Jang et al., 2009]. Ji et al. [2010] reported an exponential increase in the number of papers published annually that incorporated a drug or gene within electrospun fibers for tissue engineering approaches. Schaub et al. [2015a] recently reviewed the literature investigating the study of neural and glial cell response to electrospun fibers from in vitro studies and summarized the few studies employing electrospun fibers within animal models. Importantly, the review by Schaub et al. [2015a] noted that general fibrous physical features (alignment, density, and diameter) influence the degree and extent of neurite outgrowth.

The process of electrospinning produces fibers with nanometer or micrometer diameters. Several in-depth reviews describe the science of electrospinning [Reneker and Chun, 1996; Pham et al., 2006; Reneker and Yarin, 2008; Sill and von Recum, 2008]. Briefly, the electrospinning process relies on an electric voltage drop to draw a thin jet from a polymer-loaded capillary, which is subsequently deposited onto a grounded collector [Bhardwaj and Kundu, 2010]. The solvent used to dissolve the polymer evaporates as the jet travels through the electric field. The jet's charge distribution (a function of the chemistry of the polymer or the inclusion of other charged molecules) causes unstable twisting and whipping that exerts a straining force, drawing the jet into a thin fiber [Reneker and Chun, 1996; Reneker et al., 2000; Reneker and Yarin, 2008]. The fiber lengthens until enough solvent evaporates for the polymer chain interactions to resist the cou-

Electrospun Fibers for Drug Delivery after SCI lombic forces exerted by the electric field [Reneker et al., 2000]. Fibers are often collected on a grounded stationary plate, producing randomly organized fibers. If the fibers are specifically designed to direct extending axons, they should be collected in a manner that produces aligned, electrospun scaffolds. Several approaches are utilized to align electrospun fibers. The most common method is to align the fibers using a rotating mandrel [Gupta et al., 2009; Wang et al., 2009], but alternative approaches use a parallel plate technique [Jeffries and Wang, 2013].

Wang et al. [2009] optimized electrospun fiber parameters using a custom-made electrospinner and created highly aligned scaffolds using a $8 \mathrm{wt} \%$ PLLA polymer dissolved in chloroform, driven through a beveled 22-gauge needle tip at a flow rate of $2 \mathrm{ml} / \mathrm{h}$, and deposited onto a mandrel rotating at a rate of $1,000 \mathrm{rpm}$. The distance between the needle and the rotating collector $(22 \mathrm{~cm}$ in diameter) was fixed at $5.5 \mathrm{~cm}$, the voltage was held constant at $20 \mathrm{kV}$, and the humidity was maintained between 30 and $40 \%$. Recently, the electrospinning apparatus was further optimized to control the humidity within $1-2 \%$ because uncontrolled humidity can place surface nanotopography onto the fibers which may influence cell behavior [Schaub et al., 2013].

Previous studies using electrospun fibers for neural applications demonstrate the importance of fiber alignment and diameter for facilitating fast, directed neurite extension. Highly aligned fibers enhanced astrocyte influx into the conduit and supported robust axonal regeneration into the biomaterial in vitro and in vivo, while a scaffold with randomly organized fibers supported less astrocyte migration and axonal regeneration [Wang et al., 2009; Hurtado et al., 2011; Zuidema et al., 2014]. In vitro studies also reveal the importance of fiber diameters. Aligned fibers with diameters in the range of $1-2 \mu \mathrm{m}$ induce the most robust neurite extension compared to fibrous scaffolds consisting of fibers with diameters of less than $750 \mathrm{~nm}$ [Wang et al., 2010; Lee et al., 2012].

\section{Methods Used to Incorporate Drugs into Electrospun Fibers}

Electrospun fiber conduits provide topographical cues for cells and axons following SCI. Furthermore, the inclusion of therapeutic molecules into biomaterial scaffolds for local, sustained release increases regeneration after SCI. However, the inclusion of these molecules into electrospun fibers can alter the morphology of electrospun fibers, leading to changes in the cellular or axonal re- 
sponse. Drugs are commonly incorporated into these scaffolds by embedding them into the polymer matrix during fiber fabrication or attaching the molecules to the fiber surface. These two approaches are described in detail by Meinel et al. [2012]. Briefly, drugs are incorporated into electrospun fiber scaffolds most commonly by the following approaches: (1) adsorbing the drug to the surface of the already fabricated electrospun fibers; (2) adding the drug molecule to the polymer solution prior to electrospinning, or (3) using the method described in (2) to create a drug-loaded core which is ensheathed by a drug-free (or drug-loaded) polymer shell via a method known as coaxial electrospinning [Su et al., 2012; Qian et al., 2014]. All of these methods are used to load electrospun fiber scaffolds with drugs, and each strategy has its own strengths and weaknesses, as highlighted below.

Physical adsorption of drug molecules onto electrospun fibers is necessary when the drug employed is denatured by or is insoluble in the electrospinning solvent. The physical adsorption of a drug to the surface of the fibers leads to a burst release of the drug. A burst release may be needed to attenuate a specific cellular response (such as inflammation). Drugs requiring a sustained release (such as a growth factor) require a different inclusion method into the fibers. To eliminate a burst release, drugs may be attached to the fiber surface through covalent coupling. However, covalent coupling of a drug to the fiber surface can alter the surface topography, as recently demonstrated in a study by Schaub et al. [2015b]. Liu et al. [2012] successfully immobilized the neurotrophin NT-3 and the enzyme chondroitinase ABC (ChABC) to fibers. NT-3 promotes neurite extension and ChABC degrades chondroitin sulfate proteoglycans and can reduce the glial scar. Crosslinking of these agents reduced their burst release, and sustained release of both molecules was observed up to 28 days in vitro. Covalently coupling drugs to fiber surfaces may enhance the ability of electrospun fibers to stimulate SCI regeneration. However, to our knowledge, only one drug-containing fibrous scaffold has been tested within a rodent model of SCI [Zhu et al., 2010].

While extended drug release from fiber surfaces only occurs through covalent attachment, drug incorporation into the polymer matrix can overcome the limitations of covalent attachment (changing the topography of fibers and increased processing time). If the drug is soluble in the polymer solvent and not chemically altered by the process of electrospinning, the drug can be incorporated within the fiber using a process known as emulsion electrospinning. Schaub and Gilbert [2011] used emulsion electrospinning to incorporate a small-molecule antimetabolic drug, 6-aminonicotinamide (6AN), into electrospun PLLA fibers. 6AN incorporation into the fibers allowed for its sustained release over 14 days. The bioactivity of released $6 \mathrm{AN}$ was confirmed within astrocytic cultures, where the released 6AN significantly decreased astrocyte metabolic activity. Other labs used emulsion electrospinning to incorporate drugs into fibers for eventual SCI applications [Chew et al., 2005; Mohtaram et al., 2015]. A limitation with emulsion electrospinning is that the drug inclusion changes the fiber's physical properties, specifically fiber alignment and diameter [Zeng et al., 2003; Chew et al., 2005; Luong-Van et al., 2006; Schaub and Gilbert, 2011]. Alterations in fiber alignment and diameter affect the rate of neurite extension and cellular migration along the fibers [Wang et al., 2009, 2010; Zuidema et al., 2014].

Coaxial electrospinning places a drug within the fiber and not throughout the fiber matrix. Coaxial electrospinning overcomes situations where the drug cannot be incorporated into the electrospinning solution due to insolubility with solvent. The coaxial device pumps two polymer solutions simultaneously through separate coaxial needles. The outer solution typically consists of a polymer placed into an organic solvent, and the inner solution contains a different polymer solution mixed with the drug or the drug alone. Coaxial electrospinning is versatile because each solution can contain a different drug, allowing for the dual release of two drug molecules [Su et al., 2012]. Additionally, both the shell and core can contain the same drug to create unique release rates of the same drug [Qian et al., 2014]. By altering the polymer, the polymer concentration, or electrospinning parameters, the core and shell fibers can have variable degradation and drug release rates [Venugopal et al., 2008]. Even with the advantages of fabricating a core-sheath fiber, coaxial electrospun fibers are not currently studied within experimental models of SCI. Furthermore, coaxial electrospinning still involves organic solvents which can negatively impact the bioactivity of drugs being incorporated into fibers.

\section{Drug Inclusion during Emulsion Electrospinning Alters a Fiber's Physical Properties}

While covalent attachment of drugs to electrospun fiber surfaces and coaxial electrospinning can increase the duration of drug release, emulsion electrospinning is an easier approach because there is no postprocessing chem- 
istry, and the electrospinning apparatus is simpler. However, as previously mentioned, the incorporation of a drug into the electrospinning emulsion creates fibers with altered physical properties. The inclusion of a drug may alter solution viscosity, surface tension and conductivity, all of which regulate the morphology of electrospun fibers.

As discussed earlier, electrospinning employs an electric voltage drop to draw a thin jet from a polymer-loaded capillary. The voltage drop also helps deposit the fiber onto a grounded collector. As the electrical field strength increases, the solution at the tip of the capillary forms a Taylor cone, with the charge concentrating in the center of the cone. When a critical voltage value is reached, defined by viscosity, conductivity and surface tension of the polymer solution, the electric force overcomes the surface tension of the solution and a polymer jet is propelled from the tip of the Taylor cone toward the collector [Bhardwaj and Kundu, 2010]. Here, we refer to the jet as a fluid cylinder just emitted from the tip of the Taylor cone before the solvent evaporates to form a solid fiber. As the jet whips unstably through the electric field, the solvent evaporates, bringing the polymer chains into contact with each other. The jet lengthens and thins until enough solvent evaporates for the polymer chain interactions to resist the coulombic forces exerted by the electric field [Reneker et al., 2000]. In the following section we explain how drug inclusion may alter the fiber properties during the jet formation and the unstable whipping stage of the electrospinning process. This section is summarized in the flow chart in figure 4, which shows the relationship between electrospinning solution parameters (blue and gray boxes) and the changes in fiber morphology (green boxes). Figure 4 also lists methods to rescue the fiber morphology based on the references in this section (white boxes).

\section{Charge Carriers}

The electrospinning process is sensitive to the number of charge carriers in the electrospinning solution. The addition of a drug may decrease the fiber diameter if it increases the number of charge carriers in the solution. The increased coulombic repulsion initiates the unstable whipping stage earlier [Zong et al., 2002] and flow of the charge increases the stretching force applied to the jet. Since the fiber is thinned during the whipping stage the increased stretching force for a longer period of time decreases the final fiber diameter (fig. 4) [Zong et al., 2002; Choi et al., 2004; Bao et al., 2013; Zeng et al., 2013]. Choi et al. [2004] showed that the fiber diameter was decreased

Electrospun Fibers for Drug Delivery after SCI when ammonium salts were added to a poly(3-hydroxybutyrate-co-3-hydroxyvalerate)/chloroform electrospinning solution. Addition of the salts increased the conductivity of the solution without significantly altering the surface tension or solution viscosity. Zeng et al. [2013] confirmed the findings from Choi et al. [2004] but used volatile salts to reduce the diameter without leaving the salt ions in the fibers after electrospinning. In general, adding a charged drug to the electrospinning solution will likely decrease the diameter of the fibers relative to a nondrug control.

\section{Chain Entanglement}

The size of the drug incorporated may also influence the fiber diameter by interrupting chain entanglement and altering the solution viscosity [Shah and Shertukde, 2003; Zamani et al., 2010] (fig. 4d, e). During electrospinning, the jet is stretched until the molecules overlap and entangle enough to oppose the force induced by the electric field [Reneker et al., 2000; McKee et al., 2004; Mituppatham et al., 2004]. The critical chain entanglement, $\mathrm{C}_{\mathrm{e}}$, is the point at which there is sufficient chain overlap to form a fiber [McKee et al., 2004]. A plasticizer, in this case the drug, interrupts the contact between overlapping chains and decreases the solution viscosity. This process is also described as adding free volume. The lower viscosity solution emits a thinner jet from the Taylor cone, resulting in smaller-diameter fibers.

A larger-sized (i.e. larger hydrodynamic volume) molecule introduces more free volume into the system, and will interrupt chain entanglement to a greater extent than a smaller molecule (fig. $4 \mathrm{~d}$, e). The experimental section of this article examines how drugs of different sizes affect the resulting fiber diameter. In figure 4, riluzole and NT-3 are inserted into the appropriate columns to show how they fit into this diagram. Similarly, increasing the concentration of a drug will introduce more free volume [Dlubek et al., 2003] and should therefore decrease the fiber diameter further. To summarize these points, the more free volume that is inserted into the emulsion solution (higher drug concentration, increased drug size), the lower the viscosity of the solution (unless there are noncovalent interactions). As a result, a thinner jet is emitted from the Taylor cone, and the jet must thin further before there is sufficient chain overlap to resist the drawing force.

\section{Noncovalent Interactions}

Conversely, increased solution viscosity increases the diameter of the jet emitted from the tip of the Taylor cone 


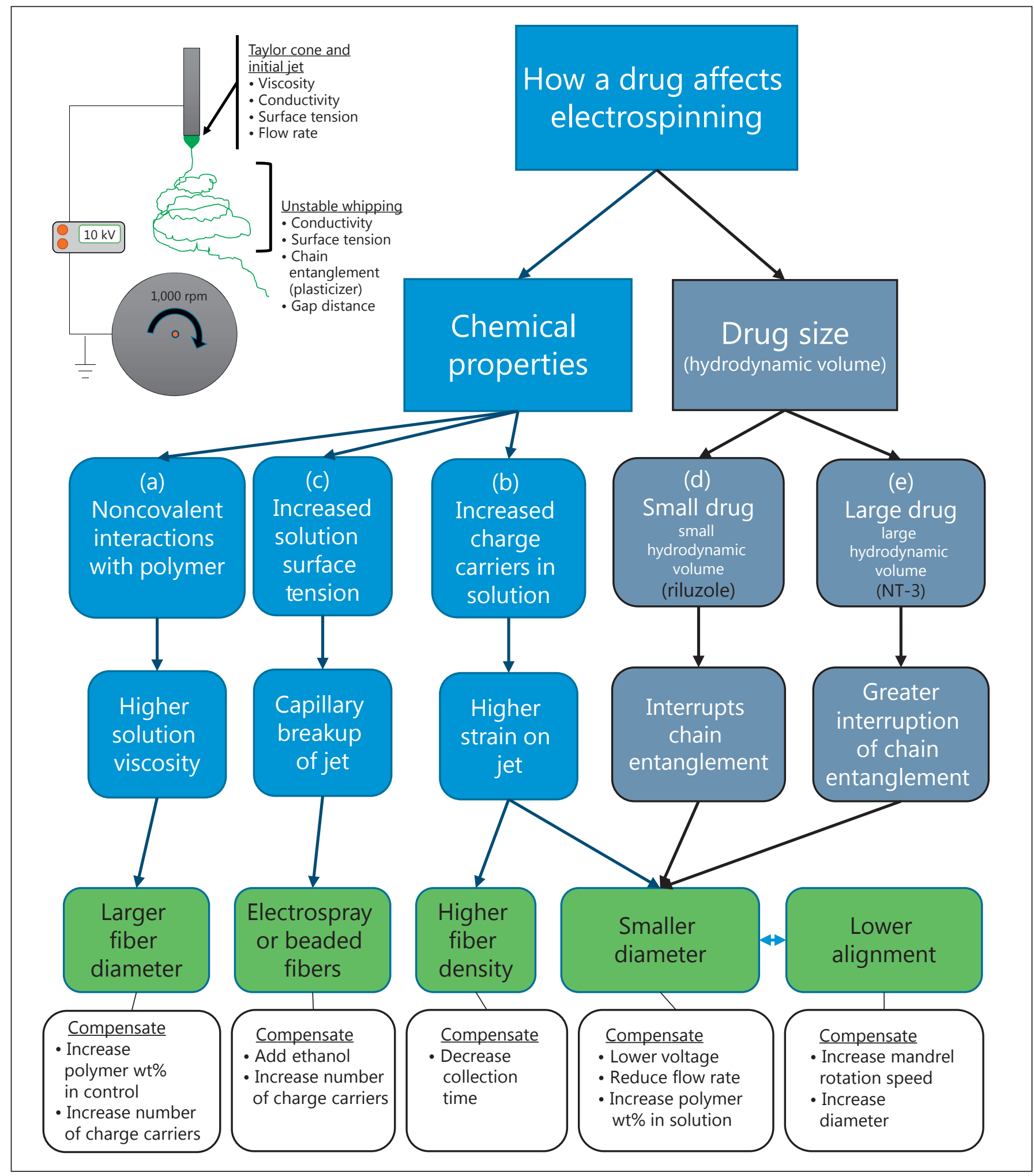

Fig. 4. The diagram and flow chart summarize the effects a drug may have on the morphology of the fibers. The diagram is a representation of the electrospinning process that highlights the Taylor cone and initial jet formation and the unstable whipping re- gion. Below each caption are the primary factors that control the fiber morphology in those regions. The flow chart links altered fiber outcomes (green boxes) with possible causes (blue and grey boxes). 
[Pham et al., 2006], and therefore increases the fiber diameter in the drug-loaded group [McKee et al., 2004; Griffin et al., 2011; Sheng et al., 2013; Ghitescu et al., 2015]. The increased viscosity is typically due to hydrogen-bonding or other noncovalent interactions. For example, Ghitescu et al. [2015] incorporated vanillic acid and other polyphenols into a poly(2-hydroxyethyl methacrylate) electrospinning solution and found that the fiber diameter increased with increasing concentrations of vanillic acid. The increases in fiber diameter were associated with increases in viscosity and were attributed to noncovalent interactions between the drug and the polymer (fig. 4, left column). A drug's chemical interactions may increase the diameter of the fibers, while the physical size of the drug may be an important variable in reducing the diameter of the fibers.

The most common method used to rescue fiber diameter is to adjust the percentage of polymer in the solution [Schaub and Gilbert, 2011; Fon et al., 2014]. Increasing the polymer weight percent increases the opportunity for chain entanglement, and therefore increases the viscosity of the solution. Alternatively, for drugs that cause an increase in fiber diameter, the weight percent of polymer in control fibers (without drugs) may be increased to match diameters observed in the experimental group. The fiber diameter can also be reduced by adding salts, increasing the voltage, decreasing the flow rate, or increasing the gap distance.

\section{Fiber Alignment}

Electrospun fiber alignment is a less studied parameter because it applies to more specialized cases. In SCI, aligned fibers have been shown to promote neurite outgrowth and faster astrocyte migration [Wang et al., 2010; Zuidema et al., 2014]. Fiber alignment is intricately related to fiber diameter. During electrospinning, the stretching and thinning forces elongate the smaller-diameter fibers to a greater extent than larger fibers. As a result, small diameter fibers must be spooled at a higher rate in order to maintain the alignment. If the rotation speed is the same, a longer spooled fiber will be less aligned than a shorter fiber. Incorporating a drug that decreases fiber diameter may reduce fiber alignment due to the increased length of the fibers.

\section{Surface Tension}

Surface tension is mainly determined by the solvent [Theron et al., 2004], but incorporating a drug may alter the surface tension of the polymer solution. Spherical shapes have lower surface energy than cylindrical jets,

Electrospun Fibers for Drug Delivery after SCI so increasing surface tension promotes beads instead of fiber formation. This tendency to bead is opposed by the coulombic repulsion and flow of charge that promotes the elongation of the jet into a cylindrical fiber [Taylor, 1964; Yarin et al., 2001; Reneker and Yarin, 2008]. The most common method for altering the surface tension is to introduce ethanol into the electrospinning solution [Fong et al., 1999; Zhang et al., 2005]. Ethanol lowers the surface tension and reduces the bead formation in poly(ethylene oxide) fibers [Fong et al., 1999; Zuo et al., 2005]. However, when a polymer was used that was not soluble in ethanol [poly(vinyl alcohol)], the phase separation had the opposite effect and fibers became beaded with added ethanol [Zhang et al., 2005]. If an introduced drug creates beaded fibers, there are two methods to rescue the smooth fiber morphology: mix solvents to reduce the surface tension [Fong et al., 1999; Zuo et al., 2005] or increase the stretching force applied to the jet by either increasing the number of charge carriers by adding a salt or increasing the voltage of the system [Reneker et al., 2000; Zhang et al., 2005; Zuo et al., 2005].

\section{System Parameters}

The system parameters can often be adjusted to rescue changes in fiber morphology. These parameters have been discussed in many reviews [Reneker et al., 2000; Pham et al., 2006; Sill and von Recum, 2008] and will only be briefly addressed here. The applied voltage affects the volume of the solution drawn from the tip of the Taylor cone. There is an operating range of applied voltage, and beaded fibers form when the voltage is either above or below this range [Sill and von Recum, 2008]. The operating range is dependent on the interaction of many of the parameters mentioned above (solvent selection, viscosity, charge carriers) and must be determined in each case. Increasing the gap distance between the needle tip and collector increases the amount of time for solvent evaporation from the fiber. Doshi and Reneker [1995] showed that fiber diameter decreased with increasing gap distance because the fiber was subjected to strain for a longer period of time. Finally, decreasing the polymer solution flow rate decreases the diameter of the resulting fibers [Fridrikh et al., 2003]. Intuitively, there is less polymer solution available at a slow pump rate for ejection from the capillary tip, which decreases the fiber diameter. Thus, if the net result of drug inclusion into fibers is a reduced fiber diameter, the applied voltage, gap distance, and solution flow rate may be adjusted to recover the fiber diameter. 
In the previous section, we discussed a number of mechanisms by which drug incorporation may affect fiber morphology. In the following experimental section, we will examine the ability of two different drugs used frequently within experimental models of SCI to alter the fiber alignment and diameter.

\section{Experimental Section}

\section{Introduction}

\section{PLLA Rationale}

High molecular weight PLLA was selected as a biomaterial because it is a long-chain aliphatic polyester. The high molecular weight increases chain entanglement and promotes fiber formation [Fong et al., 1999], and the polyester linkages are biodegradable [Uhrich et al., 1999]. PLLA is a widely used biomaterial [Uhrich et al., 1999; Drumright et al., 2000; Yang et al., 2004; Corey et al., 2008; Schaub and Gilbert, 2011; Zeng et al., 2013; Koppes et al., 2014; Zuidema et al., 2014] and is FDA approved [Palm and Goldman, 2009]. In this study, we designed the diameter of the fibers to be approximately $1 \mu \mathrm{m}$. This is based on previous studies which suggest that the optimal fiber diameter for the CNS is between 0.5 and $5 \mu \mathrm{m}$ [Wen and Tresco, 2006; Wang et al., 2010; Lee et al., 2012].

\section{Riluzole Rationale}

Riluzole is a neuroprotective agent known to block the excitotoxic effects of glutamate. Riluzole (2-amino-6-trifluoromethoxy benzothiazole) is marketed by Sanofi Pharmaceuticals under the name RILUTEK ${ }^{\circledR}$, with clinical use in patients with amyotrophic lateral sclerosis. In neurons, riluzole inhibits glutamate release, inactivates voltage-gated sodium channels, and interferes with the events that follow glutamate binding to its receptor [2012]. Riluzole also affects astrocytes. A study placed riluzole in cultures of primary rat spinal cord astrocytes and motor neurons and found that there was a 2-fold increase in motor neuron survival when compared to survival observed in L15 media control cultures [Peluffo et al., 1997]. Later studies found riluzole to improve glutamate uptake and increase growth factor synthesis within astrocytes [Frizzo et al., 2004]. Specifically, riluzole increased the production of three important neurotrophic factors: BDNF, glial cell-derived neurotrophic factor (GDNF), and nerve growth factor in mouse astrocytes [Mizuta et al., 2001]. Therefore, releasing riluzole from electrospun fibers should help reduce neuron excitotox- icity and increase the neuroprotective capacity of astrocytes in a sustained manner.

\section{NT-3 Rationale}

NT-3 is a growth factor protein that acts on neurons by binding to tyrosine kinase receptor $\mathrm{C}$ or low-affinity neurotrophin receptor p75 [Lewin and Barde, 1996; Morcuende et al., 2013]. Multiple studies have shown that neurotrophins, especially NT-3, are required to enhance axonal regeneration and neuron survival following SCI [Maisonpierre et al., 1990; Schnell et al., 1994; Mocchetti and Wrathall, 1995; Skaper, 2008], even in growth-permissive environments [Hou et al., 2012]. Multiple studies reveal the benefit of local delivery of NT-3 to the injured CNS using various delivery methods [Taylor et al., 2004, 2006; Fan et al., 2011; Yao et al., 2013; Elliott Donaghue et al., 2015]. Here, we explore a new approach for locally delivering NT-3 via electrospun fibers.

\section{Materials and Methods}

\section{Electrospinning Apparatus}

For this study, electrospun fibers were fabricated in a closed environment to control and maintain humidity which, if uncontrolled, can alter electrospun fibers surface morphology. To control humidity, the electrospinning apparatus was placed in a $35 \times$ 36-in dissipative PVC glove box (Terra Universal, Fullerton, Calif., USA). The electrospinning device contains a syringe pump (model No. R99-E; Razel Scientific, St. Albans, Vt., USA) with a 5-ml syringe containing the electrospinning solution and fitted with a 22.5-gauge needle (Becton Dickenson, Franklin Lakes, N.J., USA). The syringe pump was placed over a grounded rotating collection disc ( $1 \mathrm{~cm}$ thick and $22 \mathrm{~cm}$ in diameter), attached to an IKA Eurostar 20 variable speed motor (model No. UX-50702-35; Wilmington, N.C., USA). A high voltage power supply (model No. ES5OP10W; Gamma High Voltage Research, Ormond Beach, Fla., USA) was connected to the needle. The syringe pump was mounted on a movable platform which allows the user to alter the position of the needle during fabrication to uniformly distribute the electrospun fibers on the glass cover slips $(15 \times 15 \mathrm{~mm}$; Knittel Glass, Brausenweig, Germany).

\section{Electrospinning Fibers Containing Riluzole}

The concentration of riluzole required for release from electrospun fibers was determined based on previous studies demonstrating its efficacy [Peluffo et al., 1997; Mizuta et al., 2001; Frizzo et al., 2004]. Electrospinning solutions were prepared at a concentration of 1,000 times the therapeutic release concentration, and were prepared by dissolving 0,5 , or $10 \mathrm{mg}$ of riluzole (Sigma-Aldrich, St. Louis, Mo., USA) in $1.0 \mathrm{ml}$ of chloroform (Sigma-Aldrich). To achieve an $8 \%$ PLLA wt/wt solution, $80 \mathrm{mg}$ of PLLA (NatureWorks, Blair Nebr., USA) were then added to the chloroform solution. Solutions were shaken vigorously at $250 \mathrm{rpm}$ on a shaker plate to homogenize. After 3-5 h, the solutions were electrospun using the apparatus described above. The solutions are referred to in the 
following sections by the riluzole weight percent relative to the weight of PLLA $(0,6.25$, or $12.5 \%)$. Solutions were loaded into a 5 -ml syringe and electrospun onto $15 \times 15$-mm coverslips using the following parameters: $5-\mathrm{mm}$ gap distance, 22 -gauge syringe, $2-\mathrm{ml} / \mathrm{h}$ flow rate, and $22-30 \%$ humidity, onto a $22-\mathrm{mm}$-diameter wheel rotating at $1,000 \mathrm{rpm}$.

\section{Electrospun Fibers Containing NT-3}

Electrospun fibers containing NT-3 were fabricated using 1,1,1,3,3,3-hexafluoro-2-propanol (HFP; Sigma-Aldrich) as the organic solvent. A total of $240 \mathrm{mg}$ of PLLA (grade 201D, lot No. 905189-2; NatureWorks), which was purchased from Cargill Dow LLC (Minnetonka, Minn., USA), was added to $2 \mathrm{~g}$ of HFP to create a $12 \%$ PLLA solution. NT-3 (catalog No. 450-03, PeproTech, Rocky Hill, N.J., USA) was previously aliquoted at a concentration of $0.25 \mathrm{mg} /$ $\mathrm{ml}$ in a $1.0 \%(\mathrm{w} / \mathrm{v})$ bovine serum albumin (BSA) solution in deionized water. The highest loading of NT-3 in a solution for this study was $5 \mu \mathrm{g}$ of NT-3/240 mg PLLA, which meant that $20 \mu \mathrm{l}$ of BSA solution was added to the solution containing $5 \mu \mathrm{g}$ of NT-3. Thus, for NT-3-free control solutions, $20 \mu \mathrm{l}$ of NT-3 free 1\% BSA was added to the PLLA solution. For the lower loading capacity of NT-3 ( $1 \mu \mathrm{g}$ NT-3/240 mg PLLA) $16 \mu \mathrm{l}$ of $1 \%$ BSA was added to the PLLA solution because $4 \mu \mathrm{l}$ of the NT-3 aliquot was added to the solution. Syringes were filled with PLLA solutions and loaded to the syringe pump. Solutions were electrospun for 15 min onto glass coverslips at a collection distance of $5 \mathrm{~cm}$, an applied voltage of $10 \mathrm{kV}$, a solution flow rate of $2 \mathrm{ml} / \mathrm{h}$, and a wheel rotation speed of 1,000 rpm. The fibers were electrospun in a relative humidity range of $30-35 \%$ based on prior lab work that determined the humidity range optimal for increased fiber collection [Schaub and Gilbert, 2011].

\section{Scanning Electron Microscopy}

The fiber-coated coverslips were removed from the collection wheel and stored at room temperature until analysis. Fibers were analyzed with scanning electron microscopy (SEM) to determine the fiber alignment, diameter, and density. The fibers were first coated with a 5-nm coating of platinum using a Technics Hummer $\mathrm{V}$ sputter coater. The fibers were imaged using a Versa 3D SEM (FEI, Hillsboro, Oreg., USA) at an acceleration voltage of $2 \mathrm{kV}$ and a working distance of $10 \mathrm{~mm}$. For the riluzole fibers, five images were taken from each sample at $\times 2,500$ at evenly distributed locations on the coverslip. The same approach was used for NT-3 fibers; however, these fibers were imaged at $\times 6,500$ due to their smaller diameter.

Fiber Analysis

Images were analyzed using NIH Image J software $(\mathrm{NIH}$, Bethesda, Md., USA). The fiber diameter was analyzed by drawing a line perpendicular to the orientation of each fiber and comparing the length of that line to the length of the SEM micrograph's scale bar. The cross-sectional diameters of at least 50 individual fibers were measured for each set of electrospun fibers to obtain an average fiber diameter. The data are reported as the mean \pm standard deviation. Three independently fabricated replicates were analyzed for each fabrication condition $(n=3)$. Fiber alignment was determined by measuring the angle a fiber deviated from the primary orientation of the fibers. The primary orientation was determined as the median value of the data set. Each angle of deviation was placed into a data bin of $3^{\circ}$ to create a histogram showing the variance in fiber alignment for each fabrication condition.

Electrospun Fibers for Drug Delivery after SCI

\section{Riluzole Incorporation Analysis}

The riluzole content in electrospun fibers was determined using a fluorescamine assay (F9015, Sigma-Aldrich). To prepare the experimental samples, fibers from each treatment group were weighed using a XS64 balance (Mettler-Toledo, Columbus, Ohio, USA), then transferred to a clean glass vial and submerged in $2 \mathrm{ml}$ of $95 \%$ (v/v in water) ethanol for $24 \mathrm{~h}$ on a plate rotating at 200 $\mathrm{rpm}$. The fibers were then removed from the vials and the ethanol was allowed to evaporate, leaving the dissolved riluzole to crystallize. Each sample was resuspended in $1.0 \mathrm{ml}$ of dimethyl sulfoxide (DMSO; 41648, Sigma). The 8\% PLLA, 0\% fibers (no riluzole) served as a negative control, while a $5-\mathrm{mg} / \mathrm{ml}$ riluzole solution in ethanol was used as a positive control. The fluorescamine assay detects the free amine present in riluzole. The standard curve was prepared using serial dilutions of riluzole in DMSO, starting at 10 $\mathrm{mg} / \mathrm{ml}(10,5,2.5,1.25,0.625$, and $0.312 \mathrm{mg} / \mathrm{ml})$. A $3-\mathrm{mg} / \mathrm{ml}$ fluorescamine solution was prepared in DMSO. The fluorescamine solution was mixed thoroughly with each sample using a 1:1 dilution. The samples were incubated in the dark at room temperature for $15 \mathrm{~min}$, then $100 \mu \mathrm{l}$ was transferred to a flat-bottomed UV transparent 96-well plate (3635, Corning Inc., Corning, N.Y., USA). The plate was read with a Tecan Infinite M200 plate reader (Tecan, Männedorf, Switzerland) using an excitation wavelength of 365 $\mathrm{nm}$ and an emission wavelength of $470 \mathrm{~nm}$.

\section{NT-3 Incorporation Analysis}

To verify the incorporation of NT-3 in electrospun fibers, a Human NT-3 DuoSet ELISA (R\&D Systems, Minneapolis, Minn., USA) was used. Fiber scaffolds were preweighed then submerged in $1 \mathrm{ml}$ of ELISA reagent diluent for 3 days to allow for protein diffusion out of the electrospun fibers. After 3 days, the reagent diluent was removed from the fibers and analyzed via ELISA. The 3 -day time point was chosen arbitrarily to verify the loading of NT-3 into fibers. This time point does not reflect the expected time release profile of NT-3 from electrospun PLLA fibers.

\section{Statistical Analysis}

To determine statistical significances in the diameters, an ANOVA was performed with a post hoc Tukey's test. Groups were deemed statistically different at $\mathrm{p}<0.05$. Since alignments had nearly the same mean, the distribution of fiber alignment was more descriptive. The Brown-Forsythe test was used to compare the variance between different experimental groups. As with measuring fiber diameter, differences in fiber alignment were statistically significant at $\mathrm{p}<0.05$.

\section{Results}

\section{Riluzole Inclusion in Electrospun Fibers}

A fluorescamine assay was performed to verify that the electrospun fibers contained riluzole. The purpose of this experiment was to confirm the successful incorporation of riluzole into the electrospun fibers. Ethanol was selected because it penetrates into the fibers, dissolves riluzole, and does not quickly dissolve PLLA. We hypothesized that increased loading of riluzole into the emulsion would 


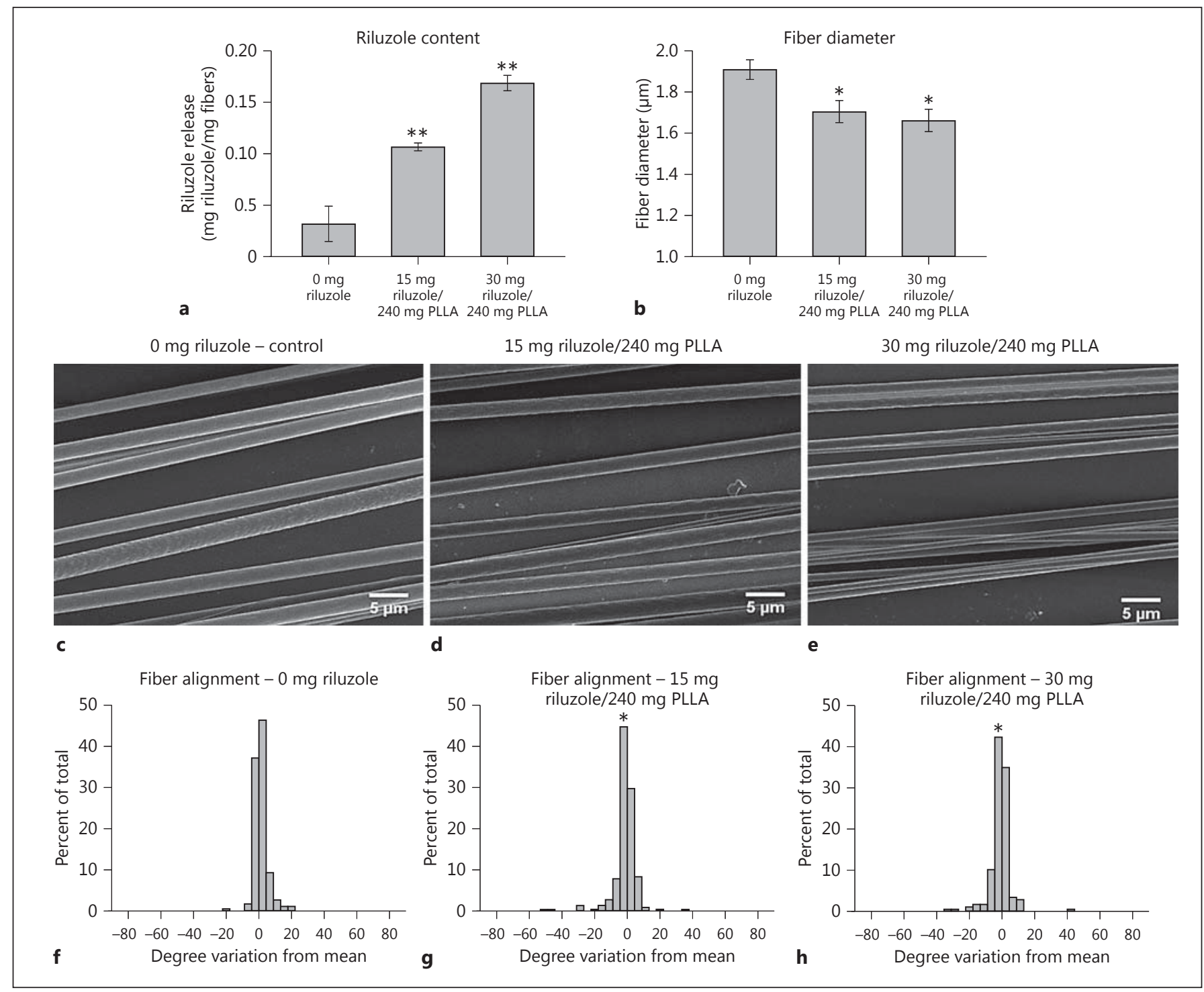

Fig. 5. Analysis of riluzole-loaded electrospun PLLA fibers. a Results of the fluorescamine assay verify successful riluzole loading. b Analysis of the fiber diameter. c-e SEM micrographs of control, $15 \mathrm{mg}$ of riluzole/240 $\mathrm{mg}$ PLLA, and $30 \mathrm{mg}$ of riluzole/240 mg PLLA fibers, respectively. $\mathbf{f}-\mathbf{h}$ Analysis of fiber alignment for control, $15 \mathrm{mg}$ of riluzole/240 $\mathrm{mg}$ PLLA, and $30 \mathrm{mg}$ of riluzole/240 mg PLLA fibers, respectively. The * indicates a significant difference

lead to an increased release of riluzole. The fluorescamine assay showed that there was a direct relationship between the two. The riluzole content from the 0,15 , and $30 \mathrm{mg}$ per $240 \mathrm{mg}$ of PLLA treatment groups respectively yielded $17.7 \pm 29.9,106.6 \pm 6.7$, and $168.6 \pm 12.8 \mu \mathrm{g}$ of riluzole per milligram of fibers (fig. 5a). Two out of the three samples from the $0-\mathrm{mg} / \mathrm{ml}$ group fell below the range of the standard curve, but their values were calculated assuming compared to the control. The ${ }^{* *}$ indicates a significant difference from all other groups (both control and experimental). An ANOVA followed by Tukey's post hoc test was used for fluorescamine assay and diameter analysis. A Brown-Forsythe test was used to describe variances in the fiber alignment $\left(*\right.$ and ${ }^{* *}$ significance $=$ $\mathrm{p}<0.05, \mathrm{n}=3$ ).

the curve extends linearly, and are reported as such. The larger degree of variability at the $0 \mathrm{mg} / \mathrm{ml}$ point may be attributed to the values being at the low end of the detection range for the fluorescamine assay.

We hypothesized that adding riluzole would decrease the diameter of the fibers since riluzole is a polar molecule. The results show that the fiber diameter decreased when riluzole was added to the electrospinning solution. 
The control fibers $(0 \mathrm{mg} / \mathrm{ml})$ had a mean fiber diameter of $1.91 \pm 0.58 \mu \mathrm{m}$. When $15 \mathrm{mg}$ of riluzole $/ 240 \mathrm{mg}$ PLLA was added to the solution, the diameter decreased by approximately $200 \mathrm{~nm}$ to $1.70 \pm 0.70 \mu \mathrm{m}$. The diameter did not significantly change when the riluzole content was raised from 15 to $30 \mathrm{mg}$ riluzole/240 mg PLLA (1.70 \pm 0.70 vs. $1.66 \pm 0.64 \mu \mathrm{m}$; fig. $5 \mathrm{~b})$, indicating that the diameter is more dependent on the presence of the small molecule rather than the concentration.

The alignment of fibers did significantly change from the control (riluzole free) when riluzole was added. The histograms of the fiber alignments display a normal distribution for the majority of the fibers (2 standard deviations) aligned to within $15^{\circ}$ of the primary axis of orientation (fig. $5 \mathrm{f}-\mathrm{h}$ ). The standard deviation of fiber alignment for 0,15 , and $30 \mathrm{mg}$ of riluzole/240 $\mathrm{mg}$ PLLA was 4.3, 7.5, and $6.5^{\circ}$, respectively. The experimental groups (15 and $30 \mathrm{mg}$ riluzole/240 mg PLLA) had more unaligned fibers that resulted in significantly different variances when compared to the control ( $0 \%$ ) using the Brown-Forsythe test $(\mathrm{p}<0.05)$.

\section{NT-3 Inclusion in Electrospun Fibers}

As seen in figure $6 \mathrm{a}$, as the amount of NT-3 added to the electrospinning solution was increased from $0 \mathrm{mg}$ to $1 \mu \mathrm{g}$ of NT-3/240 mg PLLA or $5 \mu \mathrm{g}$ of NT-3/240 mg PLLA, the amount of NT-3 released from the fibers increased. The control fibers released no NT-3 (negative values) while the electrospinning solution containing $1 \mu \mathrm{g}$ of NT$3 / 240 \mathrm{mg}$ PLLA produced fibers that released $21.4 \pm 7.6$ pg of NT-3 per mg of fibers, and the solution that contained $5 \mu \mathrm{g}$ of NT-3/240 mg PLLA produced fibers that released $30.3 \pm 9.9$ pg of NT-3 per mg of fibers over 3 days. The amounts of NT- 3 released from the $1 \mu \mathrm{g}$ of NT-3/240 $\mathrm{mg}$ PLLA and $5 \mu \mathrm{g}$ of NT-3/240 mg PLLA solution groups were not statistically different. These results confirm that the fibers made with NT-3 included in the electrospinning solution actually contained NT-3. Subsequently, the fiber diameter and alignment were assessed using SEM.

SEM micrographs were captured for each fiber group and analyzed using Fiji software to characterize the average diameter and alignment of each group of electrospun fibers. As the concentration of NT-3 in the solution was increased, there was a significant decrease in the average fiber diameter (fig. 6b). Control (NT-3-free) fibers had an average diameter of $0.74 \pm 0.01 \mu \mathrm{m}$ while fibers fabricated from solutions containing $1 \mu \mathrm{g}$ of NT-3/240 mg PLLA or $5 \mu \mathrm{g}$ of NT-3/240 mg PLLA had average diameters of 0.64 \pm 0.3 and $0.54 \pm 0.04 \mu \mathrm{m}$, respectively. These values were all statistically different.

Electrospun Fibers for Drug Delivery after SCI
SEM micrographs (fig. 6c-e) were further analyzed to determine the fiber alignment by calculating the degree variation from the mean fiber angle of 50 fibers in each replicate fiber scaffold. The resulting analysis of variances and Brown-Forsythe test results indicated that the alignments of the control, $1 \mu \mathrm{g}$ of NT-3/240 mg PLLA, and $5 \mu \mathrm{g}$ of NT-3/240 mg PLLA loaded scaffolds were all statistically different from each other (fig. $6 \mathrm{f}-\mathrm{h}$ ). The fibers fabricated from a solution containing $5 \mu \mathrm{g}$ of NT-3/240 mg PLLA were significantly less aligned than both the other groups, while the fibers fabricated from the solution with $1 \mu \mathrm{g}$ of NT-3/240 mg PLLA were more aligned but still significantly less so than the NT-3-free control fibers.

\section{Discussion}

Hydrogel biomaterial scaffolds lacking directional cues for infiltrating cells and extending axons improve recovery after SCI, but are usually only effective if the hydrogel strategy includes a mechanism to facilitate drug release. In contrast, drug-free electrospun fiber scaffolds robustly support axonal regeneration after SCI without drugs [Hurtado et al., 2011]. Fiber guidance and enhanced regeneration outcomes may be supported by the inclusion of drugs within the fibers. In this study, riluzole and NT-3 were selected as candidate drugs because they are both frequently administered within animal models of SCI. Incorporation of riluzole or NT-3 into electrospun fibers should further improve recovery after SCI when using a fibrous scaffold approach. Riluzole is a neuroprotective agent that blocks the excitotoxic effects of glutamate, increases the astrocyte uptake of glutamate, and stimulates astrocytes to produce BDNF, GDNF, and nerve growth factor [Peluffo et al., 1997; Mizuta et al., 2001; Frizzo et al., 2004]. NT-3 reduces the lesion size, prevents secondary neuronal death, and increases axonal sprouting following SCI [Bradbury et al., 1999; Liu et al., 2012; Morcuende et al., 2013]. However, to our knowledge, no laboratory has successfully incorporated riluzole or NT-3 into electrospun fibers. The inclusion of molecules within electrospun fibers can change physical characteristics. Thus, the focus of the experimental section of this manuscript was to explore changes in the electrospun fiber physical properties when riluzole (small molecule) or NT-3 (large molecule) was incorporated into the electrospun fibers.

We hypothesized that the addition of both drugs individually would decrease the fiber diameter and negatively affect the overall alignment of the fiber scaffolds. Fur-

Cells Tissues Organs 2015-16;202:116-135 129 


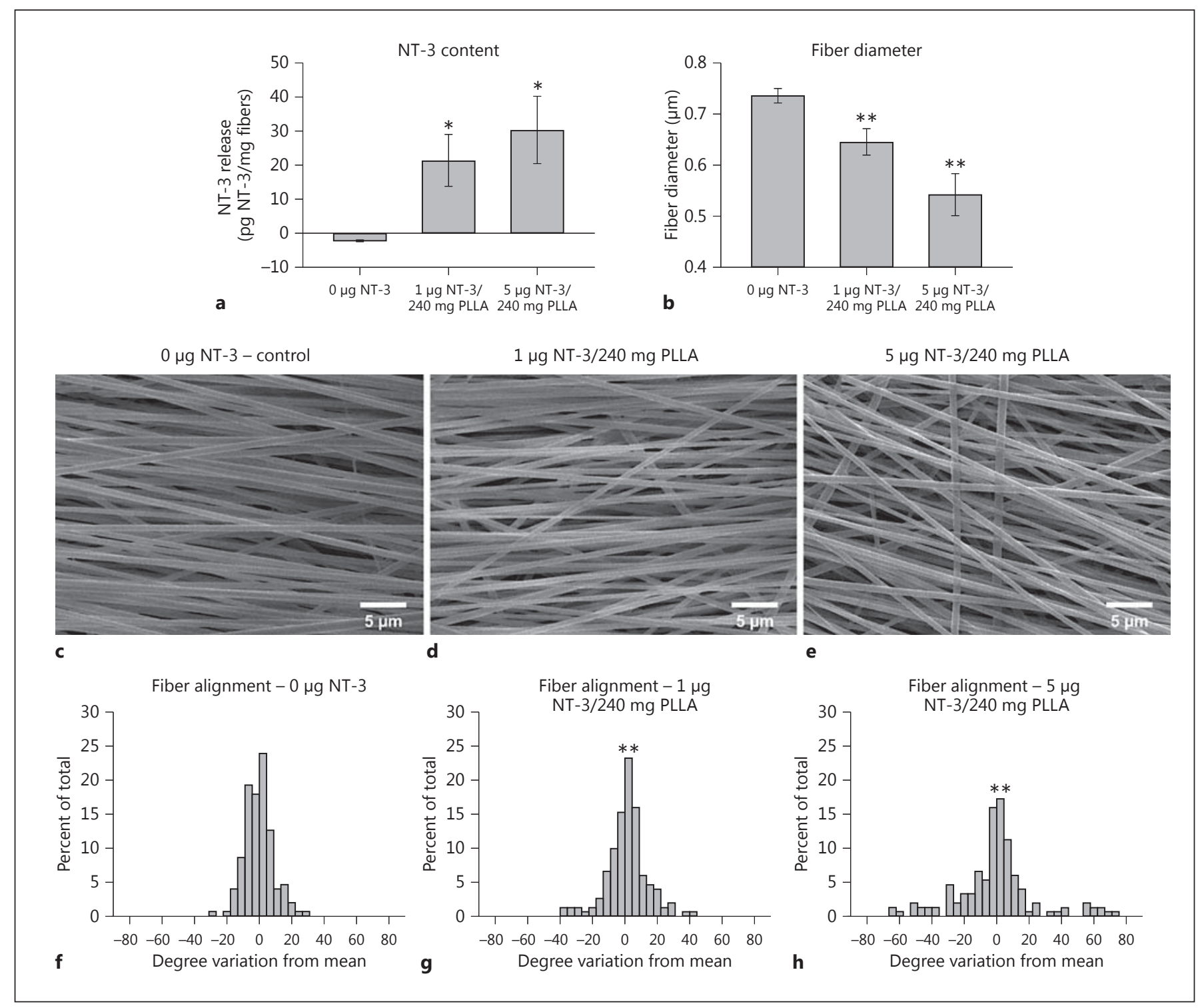

Fig. 6. Analysis of NT-3-loaded electrospun PLLA fibers. a ELISA results verifying successful NT-3 loading. b Analysis of the fiber diameter. c-e SEM micrographs of control, $1 \mu \mathrm{g}$ of NT-3/240 mg PLLA, and $5 \mu \mathrm{g}$ of NT-3/240 mg PLLA fibers, respectively. f-h Analysis of fiber alignment for control, $1 \mu \mathrm{g}$ of NT-3/240 mg PLLA, and $5 \mu \mathrm{g}$ of NT-3/240 mg PLLA fibers, respectively. The * indicates a significant difference compared to the control. The ** indicates a significant difference from all other groups (both control and experimental). ANOVA was used for ELISA and diameter analysis. A Brown-Forsythe test was used to describe variances in the fiber alignment. ( ${ }^{*}$ and ${ }^{* *}$ significance $=\mathrm{p}<0.05, \mathrm{n}=3$.). thermore, we hypothesized that these drugs would affect the fibers differently as they are both very different in size, structure, and chemistry. After verifying successful drug loading in fibers, these hypotheses were validated for both drugs by analyzing fiber morphology via SEM. The inclusion of either riluzole or NT-3 in electrospun fibers caused a reduction in fiber diameter and interfered with fiber alignment. This knowledge is important during the pre- liminary development of riluzole or NT-3-loaded electrospun fiber scaffolds as changes in electrospun fiber morphology have been shown to modify the cellular response and may introduce confounding variables into the study. For example, smaller-diameter fibers may not as efficiently support cellular migration or axonal regeneration into the lesion site and scaffolds with greater fiber crossing may impede cellular migration and axonal extension. 


\section{Diameter}

The fiber diameter was affected by the inclusion of riluzole or NT-3. When riluzole was incorporated into the electrospinning solution, the resulting fiber diameter decreased significantly by approximately $200 \mathrm{~nm}$. Similarly, after introducing NT-3 into the electrospinning solution, the resulting fiber diameter decreased. However, as the concentration of riluzole in the solution was increased, there was not a further reduction in fiber diameter. In contrast, when the amount of NT-3 in the solution was increased from $1 \mu \mathrm{g}$ of NT-3/240 mg PLLA to $5 \mu \mathrm{g}$ of NT-3/240 mg PLLA, the average fiber diameter continued to decrease. There are two likely reasons for the decrease in fiber diameter observed here: (1) the drugs act as plasticizers, interrupting chain entanglement so that the polymer jet thins to a greater extent before it is deposited [McKee et al., 2004], or (2) the drugs increase the number of charge carriers in the solution [Reneker et al., 2000; Lin et al., 2007; Schaub and Gilbert, 2011]. Increasing the charge carrier concentration causes the jet to enter the unstable whipping stage earlier and increases the stretching force on the fibers due to charge repulsion. As a result, there is more time for the fiber to thin during the whipping stage, and the fiber must become thinner before the chains can overlap to counteract the increased stretching force. Schaub and Gilbert [2011] found that small-molecule $6 \mathrm{AN}$ decreased the diameter and interrupted the alignment of fibers. To rescue this fiber diameter, the concentration of polymer in the electrospinning solution was increased, and the increased polymer concentration created fiber diameters statistically similar to the control. The same approach of increasing polymer concentration was employed when a BDNF-mimetic nonpeptide small molecule decreased the diameter of poly(L-caprolactone) fibers [Fon et al., 2014]. Thus, increasing polymer concentration would likely aid in restoring the fiber diameter after the inclusion of riluzole or NT-3. A decrease in fiber diameter is the most common effect of incorporating a drug into an electrospinning solution [Zeng et al., 2003].

The findings presented here are important because many studies show that cells in the CNS are sensitive to diameter. The unmyelinated axon caliber in the CNS typically ranges from 200 to $700 \mathrm{~nm}$ [Lee et al., 2012]. It is fitting that oligodendrocytes in the CNS myelinate electrospun fibers that are between 500 and $800 \mathrm{~nm}$ at a 5 -fold higher rate than fibers in the $300-400 \mathrm{~nm}$ range. The diameter may indicate a more mature neuron in need of myelination [Lee et al., 2012]. Wang et al. [2010] showed this same phenomenon in comparing neurite outgrowth

Electrospun Fibers for Drug Delivery after SCI from dorsal root ganglia on 300-, 750-, and 1,300-nmdiameter fibers. Their study showed that neurites extended longest on the 750- and 1,300-nm fibers [Wang et al., 2010]. Wen and Tresco [2006] prepared a series of filaments from 5 to $500 \mu \mathrm{m}$ using an extrusion process to examine the effect of fibrous scaffolds with much larger diameters. Their study showed that cells extend farther on the topography of fibers with diameters that are smaller than the cells. Neurites grew longest on the fibers that were $5 \mu \mathrm{m}$ in diameter, compared to 30-, 100-, 200-, and $500-\mu \mathrm{m}$-diameter fibers. This highlights a range of diameters between 0.5 and $5 \mu \mathrm{m}$ in which rapid migration of CNS cells was observed [Wang et al., 2010; Lee et al., 2012]. Controlling the diameter between control fibers (without drug) and experimental fibers (with drug) will better determine the role of drug inclusion and delivery influencing SCI outcomes.

\section{Alignment}

As observed in the results, in general, as the fiber diameter changes the fiber alignment is also affected. Aligned fibers promote more rapid cell infiltration into a scaffold [Wang et al., 2009; Zuidema et al., 2014]. Drug inclusion into electrospun fibers commonly affects fiber alignment by increasing jet extension. The decrease in fiber alignment that resulted from NT-3 inclusion (fig. 6b) will need to be rescued if NT-3-loaded electrospun fibers are to be viable materials for SCI applications. Zuidema et al. [2014] demonstrated that fiber misalignment can significantly impede astrocyte migration and elongation. Since the fiber scaffold should encourage cellular infiltration of astrocytes, developing procedures to rescue fiber alignment after the inclusion of NT-3 is warranted. Furthermore, Wang et al. [2009] showed that crossing fibers in electrospun fiber scaffolds can halt neurite extension from cultured dorsal root ganglia explants. Electrospun fiber scaffolds for SCI applications are placed within conduit structures to guide axonal extension across the lesion site. Thus, if crossing fibers exist within the conduit, even though a drug is present to spur faster regeneration, the crossed fibers may counteract the ability of the drug to further enhance regeneration. Wang et al. [2009] employed different electrospinning procedures to improve fiber alignment. The rotational speed of the collection mandrel had the largest impact on fiber alignment. By increasing the wheel speed from 250 to $1,000 \mathrm{rpm}$, crossing fibers were reduced. Thus, it is likely that an increase in wheel rotation speed while electrospinning NT-3 loaded PLLA fibers will result in fewer crossing fibers than those observed in figure $6 \mathrm{~d}$ and $\mathrm{e}$. 


\section{Large Molecule versus Small Molecule}

The two drugs used in this study were selected because they represent two different types of drugs studied for SCI applications. Proteins, such as NT-3 [Bradbury et al., 1999] or GDNF [Mohtaram et al., 2015], are large macromolecules that must remain bioactive despite the caustic solvent and electrospinning process. Small molecules, such as riluzole, retinoic acid, 17 $\beta$-estradiol [Cox et al., 2015], 6AN [Schaub and Gilbert, 2011], and polyphenols [Ghitescu et al., 2015], are generally more stable during the electrospinning process. Both drug classes interrupt chain entanglements by introducing free volume into the solution (reducing fiber diameter). It was hypothesized that NT-3 would have a greater effect on fiber diameter than riluzole. The larger NT-3 will likely occupy a greater amount of free volume than the compact riluzole, and therefore affect the fiber diameter more significantly. Unfortunately, due to the solubility limitations of both drugs, it is difficult to draw complete comparisons between the two. Nonetheless, there are a few trends that can be compared.

First, NT-3 had a disproportionally large effect on the fiber diameter since NT-3 was loaded at a lower concentration than riluzole. While the riluzole $(15 \mathrm{mg}$ riluzole/240 mg PLLA) loading content was approximately four orders of magnitude greater than that of NT-3 (1 $\mu \mathrm{g}$ NT-3/240 mg PLLA), the change in fiber diameter due to the drug incorporation was only different by a factor of two. The diameter of riluzole-loaded fibers was approximately $100 \mathrm{~nm}$ smaller than the corresponding control fibers, while NT-3-loaded fibers were $200 \mathrm{~nm}$ smaller in diameter than control NT-3-free fibers. This aligns with the expected differences in free volume created by NT-3 and riluzole, and supports our hypothesis that NT-3 inclusion would have a greater effect on fiber diameter than riluzole inclusion.

Second, there is noticeable peak broadening in the alignment histogram of the NT-3 results. Reduced fiber alignment is associated with a decrease in fiber diameter. The syringe pump rate and collection wheel speed were fixed across all experimental groups, and the fibers were drawn into thinner fibers with each successive experimental group. The longer, thinner fibers spooled at the same rate will be less aligned. The experimental results support this by showing that drug inclusion simultaneously decreased fiber diameter and negatively affected fiber alignment. With NT-3 fiber results, the fiber diameter significantly decreased with increasing NT-3 concentration, and the alignment also worsened with increasing NT-3 concentration. With riluzole fiber results, the dif- ferences in fiber alignment also mirrored the differences in the diameter. With the riluzole fibers, both experimental fiber groups had significantly smaller fibers compared to the controls, but there were no significant differences between the experimental groups. Both experimental fiber groups were also significantly less aligned than the control, while there were no significant differences between the experimental groups. Despite using different drugs and different solvents, the trends in alignment mirrored the changes in diameter.

\section{Conclusions}

In the experimental section of this manuscript we demonstrated that inclusion of either riluzole or NT-3 into electrospun PLLA fibers via emulsion electrospinning has significant effects on fiber physical characteristics. Specifically, we observed decreases in fiber diameter and fiber alignment resulting from the inclusion of either drug. Both drugs used in this study hold great potential in treating SCI, and this study demonstrates the first instance of including either drug into electrospun fibers. We also reviewed the potential mechanisms by which incorporation of drugs decreases fiber diameter and alignment, and suggested methods for rescuing fiber physical characteristics to optimize drug-eluting scaffolds prior to in vivo experimentation. In future work, we will experiment with rescuing the diameter of these fibers by increasing the concentration of PLLA in the solution. We will then characterize the release profiles of both riluzoleand NT-3-loaded fibers, and determine how astrocytes and neurons respond to the drug-releasing fibers in vitro.

\section{Acknowledgements}

The authors gratefully acknowledge grant support provided by the National Science Foundation (CAREER grant 11050125), National Institutes of Health (R01 NS092754), and the New York Spinal Cord Injury Research Trust (contract No. C030239) awarded to Ryan J. Gilbert, and the Predoctoral Fellowship awarded to Christopher Johnson by the New York Spinal Cord Injury Research Trust (RFA No. 1412220226). 


\section{References}

Acheson, A., J.C. Conover, J.P. Fandl, T.M. DeChiara, M. Russell, A. Thadani, et al. (1995) A BDNF autocrine loop in adult sensory neurons prevents cell death. Nature 374 : 450-453.

-Anderson, M.A., J.E. Burda, Y. Ren, Y. Ao, T.M. O’Shea, R. Kawaguchi, et al. (2016) Astrocyte scar formation aids central nervous system axon regeneration. Nature 532: 195-200.

Bao, J., W. Lv, Y. Sun, Y. Deng (2013) Electrospun antimicrobial microfibrous scaffold for annulus fibrosus tissue engineering. J Mater Sci 48: 4223-4232.

Beattie, M.S., A.A. Farooqui, J.C. Bresnahan (2000) Review of current evidence for apoptosis after spinal cord injury. J Neurotrauma 17: 915-925.

Bhardwaj, N., S.C. Kundu (2010) Electrospinning: a fascinating fiber fabrication technique. Biotechnol Adv 28: 325-347.

Bradbury, E.J., S. Khemani, R. von King, J.V. Priestley, S.B. McMahon (1999): NT-3 promotes growth of lesioned adult rat sensory axons ascending in the dorsal columns of the spinal cord. Eur J Neurosci 11: 3873-3883.

-Bresnahan, J.C. (1978) An electron-microscopic analysis of axonal alterations following blunt contusion of the spinal cord of the rhesus monkey (Macaca mulatta). J Neurol Sci 37: $59-82$.

Chew, S.Y., J. Wen, E.K.F. Yim, K.W. Leong (2005) Sustained release of proteins from electrospun biodegradable fibers. Biomacromolecules 6: 2017-2024.

Choi, J.S., S.W. Lee, L. Jeong, S.-H. Bae, B.C. Min, J.H. Youk, et al. (2004) Effect of organosoluble salts on the nanofibrous structure of electrospun poly(3-hydroxybutyrate-co-3-hydroxyvalerate). Int J Biol Macromol 34: 249-256.

-Corey, J.M., C.C. Gertz, B.-S. Wang, L.K. Birrell, S.L. Johnson, D.C. Martin, et al. (2008) The design of electrospun PLLA nanofiber scaffolds compatible with serum-free growth of primary motor and sensory neurons. Acta Biomater 4: 863-875.

Cox, A., A. Varma, J. Barry, A. Vertegel, N. Banik (2015) Nanoparticle estrogen in rat spinal cord injury elicits rapid anti-inflammatory effects in plasma, cerebrospinal fluid, and tissue. J Neurotrauma 32: 1413-1421.

Dlubek, G., V. Bondarenko, J. Pionteck, M. Supej, A. Wutzler, R. Krause-Rehberg (2003) Free volume in two differently plasticized poly(vinyl chloride)s: a positron lifetime and PVT study. Polymer 44: 1921-1926.

Doshi, J., D.H. Reneker (1995) Electrospinning process and applications of electrospun fibers. J Electrost 35: 151-160.

Drumright, R.E., P.R. Gruber, D.E. Henton (2000) Polylactic acid technology. Adv Mater 12: 1841-1846.

Elliott Donaghue, I., C.H. Tator, M.S. Shoichet (2015a) Sustained delivery of bioactive neurotrophin-3 to the injured spinal cord. Biomater Sci 3: 65-72.
Fan, J., H. Zhang, J. He, Z. Xiao, B. Chen, J. Xiaodan, et al. (2011) Neural regrowth induced by PLGA nerve conduits and neurotrophin-3 in rats with complete spinal cord transection. J Biomed Mater Res B Appl Biomater 97B: 271-277.

Faulkner, J.R., J.E. Herrmann, M.J. Woo, K.E. Tansey, N.B. Doan, M.V. Sofroniew (2004) Reactive astrocytes protect tissue and preserve function after spinal cord injury. J Neurosci 24: 2143-2155.

Fon, D., K. Zhou, F. Ercole, F. Fehr, S. Marchesan, M.R. Minter, et al. (2014) Nanofibrous scaffolds releasing a small molecule BDNF-mimetic for the re-direction of endogenous neuroblast migration in the brain. Biomaterials 35: 2692-2712.

Fong, H., I. Chun, D.H. Reneker (1999) Beaded nanofibers formed during electrospinning. Polymer 40: 4585-4592.

Fridrikh, S.V., J.H. Yu, M.P. Brenner, G.C. Rutledge (2003) Controlling the fiber diameter during electrospinning. Phys Rev Lett 90: 144502.

Frizzo M.E., L.P. Dall'Onder, K.B. Dalcin, D.O. Souza (2004) Riluzole enhances glutamate uptake in rat astrocyte cultures. Cell Mol Neurobiol 24: 123-128.

-Gelain, F., S. Panseri, S. Antonini, C. Cunha, M. Donega, J. Lowery, et al. (2011) Transplantation of nanostructured composite scaffolds results in the regeneration of chronically injured spinal cords. ACS Nano 5: 227236.

Ghitescu, R.-E., A.-M. Popa, V.I. Popa, R.M. Rossi, G. Fortunato (2015) Encapsulation of polyphenols into pHEMA e-spun fibers and determination of their antioxidant activities. Int J Pharm 494: 278-287.

Griffin, J., R. Delgado-Rivera, S. Meiners, K.E. Uhrich (2011) Salicylic acid-derived poly(anhydride-ester) electrospun fibers designed for regenerating the peripheral nervous system. J Biomed Mater Res A 97A: 230242.

Gros, T., J.S. Sakamoto, A. Blesch, L.A. Havton, M.H. Tuszynski (2010) Regeneration of longtract axons through sites of spinal cord injury using templated agarose scaffolds. Biomaterials 31: 6719-6729.

-Gupta, D., J. Venugopal, M.P. Prabhakaran, V.R.G. Dev, S. Low, A.T. Choon, et al. (2009) Aligned and random nanofibrous substrate for the in vitro culture of Schwann cells for neural tissue engineering. Acta Biomater 5: 2560-2569.

Haggerty, A.E., M. Oudega (2013) Biomaterials for spinal cord repair. Neurosci Bull 29: 445459.

He, L., Y. Zhang, C. Zeng, M. Ngiam, S. Liao, D. Quan, et al. (2009) Manufacture of PLGA multiple-channel conduits with precise hierarchical pore architectures and in vitro/vivo evaluation for spinal cord injury. Tissue Eng Part C Methods 15: 243-255.
Hou, S., L. Nicholson, E. Niekerk van, M. Motsch, A. Blesch (2012) Dependence of regenerated sensory axons on continuous neurotrophin-3 delivery. J Neurosci 32: 13206-13220.

Hurtado, A., J.M. Cregg, H.B. Wang, D.F. Wendell, M. Oudega, R.J. Gilbert, et al. (2011) Robust CNS regeneration after complete spinal cord transection using aligned poly-L-lactic acid microfibers. Biomaterials 32: 6068-6079.

-Jang, J.-H., O. Castano, H.-W. Kim (2009) Electrospun materials as potential platforms for bone tissue engineering. Adv Drug Deliv Rev 61: 1065-1083.

-Jeffries, E.M., Y. Wang (2013) Incorporation of parallel electrospun fibers for improved topographical guidance in 3D nerve guides. Biofabrication 5: 035015.

Ji, W., Y. Sun, F. Yang, J.J.J.P. van den Beucken, M. Fan, Z. Chen, et al. (2010) Bioactive electrospun scaffolds delivering growth factors and genes for tissue engineering applications. Pharm Res 28: 1259-1272.

Koppes, A.N., N.W. Zaccor, C.J. Rivet, L.A. Williams, J.M. Piselli, R.J. Gilbert, et al. (2014) Neurite outgrowth on electrospun PLLA fibers is enhanced by exogenous electrical stimulation. J Neural Eng 11: 046002.

Krishna, V., S. Konakondla, J. Nicholas, A. Varma, M. Kindy, X. Wen (2013) Biomaterial-based interventions for neuronal regeneration and functional recovery in rodent model of spinal cord injury: a systematic review. J Spinal Cord Med 36: 174-190.

Kumbar, S.G., R. James, S.P. Nukavarapu, C.T. Laurencin (2008) Electrospun nanofiber scaffolds: engineering soft tissues. Biomed Mater 3: 034002.

Lee, S., M.K. Leach, S.A. Redmond, S.Y.C. Chong, S.H. Mellon, S.J. Tuck, et al. (2012) A culture system to study oligodendrocyte myelination processes using engineered nanofibers. Nat Methods 9: 917-922.

Lewin, G.R., Y.-A. Barde (1996) Physiology of the neurotrophins. Annu Rev Neurosci 19: 289317.

Lin, K., K.-N. Chua, G.T. Christopherson, S. Lim, H.-Q. Mao (2007) Reducing electrospun nanofiber diameter and variability using cationic amphiphiles. Polymer 48: 6384-6394.

Lindsay, RM (1988) Nerve growth factors (NGF, BDNF) enhance axonal regeneration but are not required for survival of adult sensory neurons. J Neurosci 8: 2394-2405.

Liu, T., J.D. Houle, J. Xu, B.P. Chan, S.Y. Chew (2012) Nanofibrous collagen nerve conduits for spinal cord repair. Tissue Eng Part A 18: 1057-1066.

Liu, X.Z., X.M. Xu, R. Hu, C. Du, S.X. Zhang, J.W. McDonald, et al. (1997) Neuronal and glial apoptosis after traumatic spinal cord injury. J Neurosci 17: 5395-5406. 
Loo, J.A., R.R. Loo, H.R. Udseth, C.G. Edmonds, R.D. Smith (1991) Solvent-induced conformational changes of polypeptides probed by electrospray-ionization mass spectrometry. Rapid Commun Mass Spectrom RCM 5: 101105.

Luong-Van, E., L. Grøndahl, K.N. Chua, K.W. Leong, V. Nurcombe, S.M. Cool (2006) Controlled release of heparin from poly $(\varepsilon-$ caprolactone) electrospun fibers. Biomaterials 27: 2042-2050.

Macaya, D., M. Spector (2012) Injectable hydrogel materials for spinal cord regeneration: a review. Biomed Mater 7: 012001.

Maisonpierre, P.C., L. Belluscio, S. Squinto, N.Y. Ip, M.E. Furth, R.M. Lindsay, et al. (1990) Neurotrophin-3: a neurotrophic factor related to NGF and BDNF. Science 247: 14461451.

McKee, M.G., G.L. Wilkes, R.H. Colby, T.E. Long (2004) Correlations of solution rheology with electrospun fiber formation of linear and branched polyesters. Macromolecules 37: 1760-1767.

Meinel, A.J., O. Germershaus, T. Luhmann, H.P. Merkle, L. Meinel (2012) Electrospun matrices for localized drug delivery: current technologies and selected biomedical applications. Eur J Pharm Biopharm 81: 1-13.

Mit-uppatham, C., M. Nithitanakul, P. Supaphol (2004) Effects of solution concentration, emitting electrode polarity, solvent type, and salt addition on electrospun polyamide- 6 fibers: a preliminary report. Macromol Symp 216: $293-300$.

Mizuta, I., M. Ohta, K. Ohta, M. Nishimura, E. Mizuta, S. Kuno (2001) Riluzole stimulates nerve growth factor, brain-derived neurotrophic factor and glial cell line-derived neurotrophic factor synthesis in cultured mouse astrocytes. Neurosci Lett 310: 117-120.

Mocchetti, I., J.R. Wrathall (1995) Neurotrophic factors in central nervous system trauma. J Neurotrauma 12: 853-870.

Mohtaram, N.K., J. Ko, A. Agbay, D. Rattray, P.O. Neill, A. Rajwani, et al. (2015) Development of a glial cell-derived neurotrophic factor-releasing artificial dura for neural tissue engineering applications. J Mater Chem B 3: 7974-7985.

Morcuende, S., R. Muñoz-Hernández, B. BenítezTemiño, A.M. Pastor, R.R. de la Cruz (2013) Neuroprotective effects of NGF, BDNF, NT-3 and GDNF on axotomized extraocular motoneurons in neonatal rats. Neuroscience 250: $31-48$.

Norenberg, M.D., J. Smith, A. Marcillo (2004) The pathology of human spinal cord injury: defining the problems. J Neurotrauma 21: 429-440.

NSCISC (2014) Spinal cord injury (SCI) facts and figures at a glance. https://www.nscisc.uab. edu/Public/Facts\%202015.pdf.

Oyinbo, CA (2011) Secondary injury mechanisms in traumatic spinal cord injury: a nugget of this multiply cascade. Acta Neurobiol Exp (Wars) 71: 281-299.
Pakulska, M.M., B.G. Ballios, M.S. Shoichet (2012) Injectable hydrogels for central nervous system therapy. Biomed Mater 7: 024101.

Palm, M.D., M.P. Goldman (2009) Patient satisfaction and duration of effect with PLLA: a review of the literature. J Drugs Dermatol 8: S15-S20.

Peluffo, H., A. Estevez, L. Barbeito, J.M. Stutzmann (1997) Riluzole promotes survival of rat motoneurons in vitro by stimulating trophic activity produced by spinal astrocyte monolayers. Neurosci Lett 228: 207-211.

Perale, G., F. Rossi, E. Sundstrom, S. Bacchiega, M. Masi, G. Forloni, et al. (2011) Hydrogels in spinal cord injury repair strategies. ACS Chem Neurosci 2: 336-345.

Pham, Q.P., U. Sharma, A.G. Mikos (2006a) Electrospinning of polymeric nanofibers for tissue engineering applications: a review. Tissue Eng 12: 1197-1211.

Qian, W., D.-G. Yu, Y. Li, Y.-Z. Liao, X. Wang, L. Wang (2014) Dual drug release electrospun core-shell nanofibers with tunable dose in the second phase. Int J Mol Sci 15: 774-786.

Reneker, D.H., I. Chun (1996) Nanometre diameter fibres of polymer, produced by electrospinning. Nanotechnology 7: 216.

Reneker, D.H., A.L. Yarin (2008) Electrospinning jets and polymer nanofibers. Polymer 49: 2387-2425.

Reneker, D.H., A.L. Yarin, H. Fong, S. Koombhongse (2000) Bending instability of electrically charged liquid jets of polymer solutions in electrospinning. J Appl Phys 87: 4531.

Schaub, N.J., C.L. Beux, J. Miao, R.J. Linhardt, J.G. Alauzun, D. Laurencin, et al. (2015b) The effect of surface modification of aligned poly-Llactic acid electrospun fibers on fiber degradation and neurite extension: e0136780. PLoS One 10 e0136780.

Schaub, N.J., T. Britton, R. Rajachar, R.J. Gilbert (2013) Engineered nanotopography on electrospun PLLA microfibers modifies RAW 264.7 cell response. ACS Appl Mater Interfaces 5: 10173-10184.

Schaub, N.J., R.J. Gilbert (2011) Controlled release of 6-aminonicotinamide from aligned, electrospun fibers alters astrocyte metabolism and dorsal root ganglia neurite outgrowth. J Neural Eng 8: 046026.

Schaub, N.J., C.D. Johnson, B. Cooper, R.J. Gilbert (2015a) Electrospun fibers for spinal cord injury research and regeneration. J Neurotrauma DOI: 10.1089/neu.2015.4165.

Schnell, L., R. Schneider, R. Kolbeck, Y.-A. Barde, M.E. Schwab (1994) Neurotrophin-3 enhances sprouting of corticospinal tract during development and after adult spinal cord lesion. Nature 367: 170-173.

-Shah, B.L., V.V. Shertukde (2003) Effect of plasticizers on mechanical, electrical, permanence, and thermal properties of poly(vinyl chloride). J Appl Polym Sci 90: 3278-3284.
Sheng, X., L. Fan, C. He, K. Zhang, X. Mo, H. Wang (2013) Vitamin E-loaded silk fibroin nanofibrous mats fabricated by green process for skin care application. Int J Biol Macromol 56: 49-56.

Sill, T.J., H.A. von Recum (2008) Electrospinning: applications in drug delivery and tissue engineering. Biomaterials 29: 1989-2006.

Skaper, S.D. (2008) The biology of neurotrophins, signalling pathways, and functional peptide mimetics of neurotrophins and their receptors. CNS Neurol Disord Drug Targets 7: 4662.

Sofroniew, MV (2009) Molecular dissection of reactive astrogliosis and glial scar formation. Trends Neurosci 32: 638-647.

Sofroniew, M.V., H.V. Vinters (2010) Astrocytes: biology and pathology. Acta Neuropathol (Berl) 119: 7-35.

-Stokols, S., J. Sakamoto, C. Breckon, T. Holt, J. Weiss, M.H. Tuszynski (2006) Templated agarose scaffolds support linear axonal regeneration. Tissue Eng 12: 2777-2787.

$\rightarrow$ Straley, K.S., C.W. Po Foo, S.C. Heilshorn (2010) Biomaterial design strategies for the treatment of spinal cord injuries. J Neurotrauma 27: 1-19.

-Su, Y., Q. Su, W. Liu, G. Jin, X. Mo, S. Ramakrishn (2012) Dual-drug encapsulation and release from core-shell nanofibers. J Biomater Sci Polym Ed 23: 861-871.

Taylor, G (1964) Disintegration of water drops in an electric field. Proc R Soc Lond Ser Math Phys Sci 280: 383-397.

Taylor, S.J., J.W. McDonald III, S.E. SakiyamaElbert (2004) Controlled release of neurotrophin-3 from fibrin gels for spinal cord injury. J Control Release 98: 281-294.

Taylor, S.J., E.S. Rosenzweig, J.W. McDonald III, S.E. Sakiyama-Elbert (2006) Delivery of neurotrophin-3 from fibrin enhances neuronal fiber sprouting after spinal cord injury. J Control Release 113: 226-235.

Theron, S.A., E. Zussman, A.L. Yarin (2004) Experimental investigation of the governing parameters in the electrospinning of polymer solutions. Polymer 45: 2017-2030.

Tsintou, M., K. Dalamagkas, A.M. Seifalian (2015) Advances in regenerative therapies for spinal cord injury: a biomaterials approach. Neural Regen Res 10: 726-742.

Uhrich, K.E., S.M. Cannizzaro, R.S. Langer, K.M. Shakesheff (1999) Polymeric systems for controlled drug release. Chem Rev 99: 31813198.

Venugopal, J., S. Low, A.T. Choon, S. Ramakrishna (2008) Interaction of cells and nanofiber scaffolds in tissue engineering. J Biomed Mater Res B Appl Biomater 84B: 34-48.

Wang, H.B., M.E. Mullins, J.M. Cregg, A. Hurtado, M. Oudega, M.T. Trombley, et al. (2009) Creation of highly aligned electrospun poly-L-lactic acid fibers for nerve regeneration applications. J Neural Eng 6: 016001. 
Wang, H.B., M.E. Mullins, J.M. Cregg, C.W. McCarthy, R.J. Gilbert (2010) Varying the diameter of aligned electrospun fibers alters neurite outgrowth and Schwann cell migration. Acta Biomater 6: 2970-2978.

Wen, X., P.A. Tresco (2006) Effect of filament diameter and extracellular matrix molecule precoating on neurite outgrowth and Schwann cell behavior on multifilament entubulation bridging device in vitro. J Biomed Mater Res A 76A: 626-637.

-Yang, F., R. Murugan, S. Ramakrishna, X. Wang, Y.-X. Ma, S. Wang (2004) Fabrication of nano-structured porous PLLA scaffold intended for nerve tissue engineering. Biomaterials 25: 1891-1900.

Yao, L., W. Daly, B. Newland, S. Yao, W. Wang, B.K.K. Chen, et al. (2013) Improved axonal regeneration of transected spinal cord mediated by multichannel collagen conduits functionalized with neurotrophin-3 gene. Gene Ther 20: 1149-1157.
Yarin, A.L., S. Koombhongse, D.H. Reneker (2001) Taylor cone and jetting from liquid droplets in electrospinning of nanofibers. J Appl Phys 90: 4836-4846.

Zamani, M., M. Morshed, J. Varshosaz, M. Jannesari (2010) Controlled release of metronidazole benzoate from poly $\varepsilon$-caprolactone electrospun nanofibers for periodontal diseases. Eur J Pharm Biopharm 75: 179-185.

Zeng, J., H. Haoqing, A. Schaper, J.H. Wendorff, A. Greiner (2013) Poly-L-lactide nanofibers by electrospinning - influence of solution viscosity and electrical conductivity on fiber diameter and fiber morphology. e-Polymers 3: 102-110.

Zeng, J., X. Xu, X. Chen, Q. Liang, X. Bian, L. Yang, et al. (2003) Biodegradable electrospun fibers for drug delivery. J Control Release 92: 227-231.

Zhang, C., X. Yuan, L. Wu, Y. Han, J. Sheng (2005) Study on morphology of electrospun poly(vinyl alcohol) mats. Eur Polym J 41: 423-432.
Zhu, Y., A. Wang, W. Shen, S. Patel, R. Zhang, W.L. Young, et al. (2010) Nanofibrous patches for spinal cord regeneration. Adv Funct Mater 20: 1433-1440.

Zong, X., K. Kim, D. Fang, S. Ran, B.S. Hsiao, B. Chu (2002) Structure and process relationship of electrospun bioabsorbable nanofiber membranes. Polymer 43: 4403-4412.

Zuidema, J.M., M.C. Hyzinski-García, K. Van Vlasselaer, N.W. Zaccor, G.E. Plopper, A.A. Mongin, et al. (2014) Enhanced GLT-1 mediated glutamate uptake and migration of primary astrocytes directed by fibronectin-coated electrospun poly-L-lactic acid fibers. Biomaterials 35: 1439-1449.

Zuo, W., M. Zhu, W. Yang, H. Yu, et al. (2005) Experimental study on relationship between jet instability and formation of beaded fibers during electrospinning. Polym Eng Sci 45: 704-709. 